\title{
Dynamics of the Bose-Einstein condensation: analogy with the collapse dynamics of a classical self-gravitating Brownian gas
}

\author{
Julien Sopik, Clément Sire and Pierre-Henri Chavanis \\ Laboratoire de Physique Théorique (UMR 5152 du CNRS), Université Paul Sabatier \\ 118, route de Narbonne, 31062 Toulouse Cedex 4, France \\ E-mail : Sopik/Clement.Sire/Chavanis@irsamc.ups-tlse.fr
}

\begin{abstract}
We consider the dynamics of a gas of free bosons within a semi-classical Fokker-Planck equation for which we give a physical justification. In this context, we find a striking similarity between the Bose-Einstein condensation in the canonical ensemble, and the gravitational collapse of a gas of classical self-gravitating Brownian particles. The paper is mainly devoted to the complete study of the Bose-Einstein "collapse" within this model. We find that at the Bose-Einstein condensation temperature $T_{c}$, the chemical potential $\mu(t)$ vanishes exponentially with a universal rate that we compute exactly. Below $T_{c}$, we show analytically that $\sqrt{\mu(t)}$ vanishes linearly in a finite time $t_{\text {coll }}$. After $t_{\text {coll }}$, the mass of the condensate grows linearly with time and saturates exponentially to its equilibrium value for large time. We also give analytical results for the density scaling functions, for the corrections to scaling, and for the exponential relaxation time. Finally, we find that the equilibration time (above $T_{c}$ ) and the collapse time $t_{\text {coll }}$ (below $T_{c}$ ), both behave like $-T_{c}^{-3} \ln \left|T-T_{c}\right|$, near $T_{c}$.
\end{abstract}




\section{INTRODUCTION}

The Bose-Einstein condensation is a fundamental result of quantum statistics 1]. Below a critical temperature $T_{c}$, a finite fraction of bosons enters the ground state and a BoseEinstein condensate (BEC) forms [2]. Observation of Bose-Einstein condensation in cold atomic samples has been first reported in 1995 by three different groups [3, 4, 5] in a vapor of spin-polarized ${ }^{87} \mathrm{Rb},{ }^{7} \mathrm{Li}$ and ${ }^{23} \mathrm{Na}$ atoms. Apart from laboratory experiments, another interesting application of Bose condensation is related to the problem of boson star formation from the dark matter in the Universe 6]. In that case, Bose star formation involves the axion as a dark matter particle candidate [7].

Since its discovery [8, 9], several authors have attempted to develop kinetic models to describe the dynamical process of the Bose-Einstein condensation. The dynamical evolution of a homogeneous gas of bosons interacting only via "collisions" can be studied using an appropriate form of the Boltzmann equation [10, 11, 12] which takes into account the specificities of the Bose statistics. Since the gas is isolated and the energy conserved, this model describes a microcanonical situation. For this model, the Bose-Einstein condensation in momentum space has been considered by Semikoz \& Tkachev [13] and Lacaze et al. [14]. Alternatively, the canonical evolution of a system of non interacting bosons coupled to a thermostat imposing the temperature can be described by a semi-classical Fokker-Planck equation [15, 16, 17]. For $T>T_{c}$, where $T_{c}$ is the critical temperature, this equation converges towards the Bose-Einstein distribution. The main purpose of this paper is a complete study of this equation below the critical temperature $T_{c}$, in order to understand the dynamics of the formation of the condensate. Our study is restricted to a spatially homogeneous system without interaction at arbitrary temperature. Alternatively, the collapsing dynamics of a trapped Bose-Einstein condensate (BEC) with attractive interaction is often analyzed in terms of the Gross-Pitaevskii (GP) or nonlinear Schrödinger equation (see, for instance [18]). This describes the formation of a spatially inhomogeneous condensate at $T=0$. The GP equation can display a self-similar collapse, but it occurs in position space while the system that we shall consider is spatially homogeneous and the condensation occurs in $\mathbf{k}$-space.

We thus consider a population of free bosons in $d$ dimensions with dispersion relation $\varepsilon(\mathbf{k})=\frac{k^{2}}{2 m}$ (we set $m=1$ in the following). This system is assumed to be strongly coupled to a thermal bath at temperature $T$. Initially, at $t=0$, the system is prepared in an initial 
state which can be, for instance, the equilibrium distribution at some high temperature $T_{0} \gg T$. At infinite time, we expect the system to reach thermal equilibrium at temperature $T$, characterized by the Bose-Einstein distribution if $T>T_{c}$ (where $T_{c}$ is the Bose-Einstein condensation temperature)

$$
\rho_{B E}(\mathbf{k})=\frac{1}{\exp \left(\frac{\beta k^{2}}{2}+\mu\right)-1},
$$

where $\mu$ is the chemical potential. Below $T_{c}$, the equilibrium distribution is the Bose-Einstein distribution with $\mu=0$ plus a Dirac peak at $\mathbf{k}=\mathbf{0}$ (the condensate) containing the rest of the mass.

\section{A. Bosonic Fokker-Planck Equation}

In this paper, we are interested in the temporal evolution of the occupation number at momentum $\mathbf{k}$, that we call $\rho(\mathbf{k}, t)$, from an arbitrary initial state to the final equilibrium state described above. If our particles were classical instead of bosons (of course no condensate can appear in that case), the coupling to the thermal bath can be modelized by a random force and a friction, and the evolution equation for the momentum of a given particle is described by the Langevin dynamics

$$
\frac{d \mathbf{k}}{d t}=-\frac{\mathbf{k}}{\xi}+\mathbf{f}(t)
$$

where $\mathbf{f}(t)$ is a delta-correlated random force, whose components satisfy

$$
\left\langle f_{i}(t) f_{j}\left(t^{\prime}\right)\right\rangle=2 D \delta_{i j} \delta\left(t-t^{\prime}\right), \quad i, j \in[1, \ldots, d]
$$

In order to recover the equilibrium equipartition theorem, we must impose the Einstein relation

$$
D=\frac{T}{\xi}
$$

where we have set the Boltzmann constant $k_{B}=1$. The Fokker-Planck equation describing the temporal evolution of the momentum distribution reads

$$
\frac{\partial \rho}{\partial t}=\frac{1}{\xi} \nabla_{\mathbf{k}}\left[T \nabla_{\mathbf{k}} \rho+\rho \mathbf{k}\right]
$$

Ultimately, the momentum distribution converges to the expected Boltzmann distribution

$$
\rho_{B}(\mathbf{k})=Z^{-1} \exp \left(-\frac{\beta k^{2}}{2}\right) \text {. }
$$


Now, coming back to the Bose gas, we ask whether a stochastic dynamics can be introduced, which accurately describes the actual evolution [48]. Restraining ourselves to non interacting bosons, we shall see that one can give a reasonable answer to this question. This will permit the description of the dynamical formation of the condensate at and below $T_{c}$, which is the main purpose of this paper.

Several authors have considered this problem [16, 17]. Let us introduce a stochastic dynamics, by defining the rate at which particles with momentum $\mathbf{k}$ get a new momentum $\mathbf{k}^{\prime}$. Following Kaniadakis et al. [16], we assume the following form of this rate

$$
W\left(\mathbf{k} \rightarrow \mathbf{k}^{\prime}\right)=w\left(\mathbf{k}, \mathbf{k}-\mathbf{k}^{\prime}\right) a[\rho(\mathbf{k}, t)] b\left[\rho\left(\mathbf{k}^{\prime}, t\right)\right]
$$

Contrary to classical dynamics, we assume a dependence of $W\left(\mathbf{k} \rightarrow \mathbf{k}^{\prime}\right)$ with $\rho(\mathbf{k}, t)$ and $\rho\left(\mathbf{k}^{\prime}, t\right)$. Otherwise, we would simply recover the Fokker-Planck equation Eq. (5), as will be shown below. In fact, $W\left(\mathbf{k} \rightarrow \mathbf{k}^{\prime}\right)$ being a rate of departure from the state of momentum $\mathbf{k}$, it seems reasonable to assume that it should not depend on the population of this initial state. Hence, we set $a(\rho)=1$. Then, one can write the master equation describing the time evolution of the particle distribution as

$$
\frac{\partial \rho}{\partial t}=\int\left[\rho\left(\mathbf{k}^{\prime}, t\right) W\left(\mathbf{k}^{\prime} \rightarrow \mathbf{k}\right)-\rho(\mathbf{k}, t) W\left(\mathbf{k} \rightarrow \mathbf{k}^{\prime}\right)\right] d \mathbf{k}^{\prime}
$$

Assuming that the evolution is sufficiently slow, and local, such that the dynamics only permits values of $\mathbf{k}^{\prime}$ close to $\mathbf{k}$, one can develop $W\left(\mathbf{k} \rightarrow \mathbf{k}^{\prime}\right)$ in powers of $\mathbf{k}-\mathbf{k}^{\prime}$ in Eq. (8) After some algebra, and proceeding along the line of [16], one obtains a Fokker-Planck-like equation

$$
\frac{\partial \rho}{\partial t}=\nabla_{\mathbf{k}}\left[b(\rho) \nabla_{\mathbf{k}}(D \rho)+\rho b(\rho) \mathbf{J}-D \rho b^{\prime}(\rho) \nabla_{\mathbf{k}} \rho\right],
$$

where assuming isotropy, we obtain

$$
\begin{aligned}
D(\mathbf{k}) & =\frac{1}{2 d} \int w(\mathbf{k}, \Delta \mathbf{k})(\Delta \mathbf{k})^{2} d(\Delta \mathbf{k}), \\
\mathbf{J}(\mathbf{k}) & =-\int w(\mathbf{k}, \Delta \mathbf{k}) \Delta \mathbf{k} d(\Delta \mathbf{k}) .
\end{aligned}
$$

We now make the simplification that the diffusion coefficient $D(\mathbf{k})$ is momentum-independent, and that the current $\mathbf{J}$ is simply proportional to the particles velocity or momentum k:

$$
\begin{aligned}
D(\mathbf{k}) & =\frac{T}{\xi}, \\
\mathbf{J}(\mathbf{k}) & =\frac{\mathbf{k}}{\xi} .
\end{aligned}
$$


Going from Eq. (10) to Eq. (12) is a standard assumption in kinetic theory. It is exactly achieved when $w(\mathbf{k}, \Delta \mathbf{k})$ is a symmetric analytic function of $(\Delta \mathbf{k}+\mathbf{k} \Delta t / \xi) / \sqrt{\Delta t}$, where $\Delta t$ is the discretized time step (ultimately $\Delta t \rightarrow 0$ ). This function must also decay fast enough so that its two first moments are well defined. In the case of the usual Brownian motion based on the Langevin equations (2), this function is simply a Gaussian. Using Eqs. (12) and (13), Eq. (9) becomes

$$
\frac{\partial \rho}{\partial t}=\frac{1}{\xi} \nabla_{\mathbf{k}}\left[T\left(b(\rho)-\rho b^{\prime}(\rho)\right) \nabla_{\mathbf{k}} \rho+\rho b(\rho) \mathbf{k}\right] .
$$

Note that choosing $b(\rho)=1$, i.e. a transition rate which does not depends on the population of the new momentum state, leads to the standard Fokker-Planck equation Eq. (5) for classical particles. In the general case, the stationary distribution for $\mathbf{k} \neq \mathbf{0}$ satisfies

$$
\left(\frac{1}{\rho}-\frac{b^{\prime}(\rho)}{b(\rho)}\right) \nabla_{\mathbf{k}} \rho=-\beta \mathbf{k},
$$

which can be integrated, leading to

$$
\frac{\rho}{b(\rho)}=\exp \left(-\frac{\beta k^{2}}{2}-\mu\right),
$$

where $\mu$ is an integration constant. In order to recover the Bose-Einstein distribution Eq. (10), we must make the unique choice

$$
b(\rho)=1+\rho .
$$

We conclude that the classical rate is amplified by the factor $b(\rho)>1$, which translates the tendency of bosons to fill already occupied states. Note that the choice $b(\rho)=1-\rho$ leads to the Fermi-Dirac distribution [17], where $b(\rho)$ now suppresses the probability to hop to an already occupied state, as can be expected for fermions (note that a generalized FokkerPlanck equation incorporating an exclusion principle has been introduced independently in 27] in the very different context of the violent relaxation of collisionless stellar systems and $2 \mathrm{D}$ vortices). This approach has been extended to the case of intermediate statistics, associated to $b(\rho)=1+\kappa \rho(\kappa \in[-1,1])$ [17]. It can be also generalized in order to recover Tsallis statistical thermodynamics at equilibrium from a general kinetic equation [17, 28].

Focusing on the bosonic case, we finally obtain the associated Fokker-Planck equation

$$
\frac{\partial \rho}{\partial t}=\frac{1}{\xi} \nabla_{\mathbf{k}}\left[T \nabla_{\mathbf{k}} \rho+\rho(1+\rho) \mathbf{k}\right]
$$


This bosonic Kramers equation could be directly obtained from a modified Langevin equation

$$
\frac{d \mathbf{k}}{d t}=-\frac{\mathbf{k}}{\xi}(1+\rho(\mathbf{k}, t))+\mathbf{f}(t)
$$

where the friction term which tends to move the momentum toward $\mathbf{k}=\mathbf{0}$ now increases with the occupation number at momentum $\mathbf{k}$. In this context, the origin of the Bose-Einstein instability is quite clear.

Finally, for later reference, we write the bosonic Fokker-Planck equation for a spherically symmetric solution. Setting $\xi=1$, we get:

$$
\frac{\partial \rho}{\partial t}=T\left(\frac{\partial^{2} \rho}{\partial k^{2}}+\frac{d-1}{k} \frac{\partial \rho}{\partial k}\right)+d \rho(1+\rho)+k(2 \rho+1) \frac{\partial \rho}{\partial k} .
$$

For the integrated density

$$
M(k, t)=\int_{0}^{k} \rho\left(k^{\prime}, t\right) k^{\prime d-1} d k^{\prime}
$$

we obtain

$$
\frac{\partial M}{\partial t}=T\left(\frac{\partial^{2} M}{\partial k^{2}}-\frac{d-1}{k} \frac{\partial M}{\partial k}\right)+k \frac{\partial M}{\partial k}\left(\frac{1}{k^{d-1}} \frac{\partial M}{\partial k}+1\right) .
$$

It has to be noted that the bosonic Fokker-Planck equation (18) valid for non-interacting bosons in contact with a heat bath (canonical ensenble) is the counterpart of the bosonic Boltzmann equation [10, 11, 12] valid for an isolated system of interacting bosons (microcanonical ensemble). These kinetic equations are semi-classical equations where quantum mechanics enters only through the Bose-Einstein statistics. The bosonic Boltzmann equation can be derived from a fully quantum treatment as discussed in Sec. VI The justification of the bosonic Fokker-Planck equation from a fully quantum mechanics treatment would be interesting but is beyond the scope of this paper.

\section{B. Analogy with a self-gravitating gas of Brownian particles}

In a series of recent papers [28, 29, 30, 31, 32], two of the present authors have introduced and systematically studied the dynamical properties of a self-gravitating gas of Brownian particles in all spatial dimensions. This is the canonical version of the original and certainly more challenging problem of self-gravitating Newtonian particles in the microcanonical ensemble. In the overdamped limit, the Langevin equation for the position in real space of a particle reads

$$
\frac{d \mathbf{r}}{d t}=-\frac{\nabla \Phi}{\xi}+\mathbf{f}(t)
$$


where the gravitational potential $\Phi(\mathbf{r}, t)$ must be computed self-consistently using the Poisson equation

$$
\Delta \Phi(\mathbf{r}, t)=S_{d} G \rho(\mathbf{r}, t),
$$

where $S_{d}$ is the surface of the unit $d$-dimensional sphere, and $G$ is Newton's constant. The associated Fokker-Planck-Poisson (or Smoluchowski-Poisson) system is obtained straightforwardly

$$
\begin{aligned}
\frac{\partial \rho}{\partial t} & =\frac{1}{\xi} \nabla[T \nabla \rho+\rho \nabla \Phi], \\
\Delta \Phi & =S_{d} G \rho .
\end{aligned}
$$

As discussed in [33], these equations also describe the chemotaxis of bacterial populations in biology, by a proper re-interpretation of the parameters. From now on, we get rid of non essential constants by setting

$$
G=\xi=S_{d}=1 .
$$

For a time-dependent radial solution, the system of equations Eqs. (25126) can be put into a unique equation 30 ]

$$
\frac{\partial \rho}{\partial t}=T\left(\frac{\partial^{2} \rho}{\partial r^{2}}+\frac{d-1}{r} \frac{\partial \rho}{\partial r}\right)+\rho^{2}+\frac{M}{r^{d-1}} \frac{\partial \rho}{\partial r},
$$

where

$$
M(r, t)=\int_{0}^{r} \rho\left(r^{\prime}\right) r^{\prime d-1} d r^{\prime},
$$

is the integrated density. Actually, the equation for $M(r, t)$ looks even simpler

$$
\frac{\partial M}{\partial t}=T\left(\frac{\partial^{2} M}{\partial r^{2}}-\frac{d-1}{r} \frac{\partial M}{\partial r}\right)+\frac{M}{r^{d-1}} \frac{\partial M}{\partial r} .
$$

It is clear that Eqs. (28130) are strikingly similar to the dynamical equations found in the context of the Bose-Einstein condensation Eqs. (20122). Apart from the identical diffusion term, we note that the non linear terms have the same dimension as $\rho^{2}$ in Eqs. (20128) and dimension $M^{2} \times(k, r)^{-d}$ in Eqs. (2230) (we shall see later that the +1 term at the end of Eq. (22) is essentially irrelevant as far as the dynamics of the Bose-Einstein condensation at or below $T_{c}$ is concerned).

Before summarizing our results concerning the dynamics of a gas of bosons in $d=3$ described by Eqs. (2022), we would like to mention only the main results obtained for a self-gravitating Brownian gas obeying Eqs. (2830). The comparison with this surprisingly close model will certainly prove interesting. 
- In $d \geq 3$, Eqs. (2830) reproduce the known equilibrium profile for $T \geq T_{c}$. For $T<T_{c}$, a dynamical instability arises coinciding with the absence of minimum of the free energy (thermodynamical instability) [29, 30].

- In $d \geq 3$, for $T<T_{c}$, the density develops a scaling profile $\rho(r, t)=\rho_{0} f\left(r / r_{0}\right)$, with $\rho_{0}(t)=\operatorname{Tr}_{0}^{-2}(t) \sim\left(t_{\text {coll }}-t\right)^{-1}$ and $f(x) \sim x^{-2}$ for $x \rightarrow+\infty$. Hence, the central density diverges in a finite time $t_{\text {coll }}$. Note that $f$ can be calculated analytically in all dimensions, and that corrections to scaling have been evaluated [29, 30].

- In $d \geq 3$, for $T<T_{c}$, and for $t \geq t_{\text {coll }}$, the central density is swallowed by an emerging condensate at $\mathbf{r}=\mathbf{0}$, which grows like $M_{0}(t) \sim\left(t-t_{\text {coll }}\right)^{d / 2-1}$. In this post-collapse regime, the residual density obeys a backward dynamical scaling, $\rho(r, t)=\rho_{0} g\left(r / r_{0}\right)$, with $\rho_{0}(t)=\operatorname{Tr}_{0}^{-2}(t) \sim\left(t-t_{\text {coll }}\right)^{-1}$ and $g(x) \sim x^{-2}$ for $x \rightarrow+\infty$. For large time, the central condensate saturates exponentially to the total mass, whereas the residual density vanishes exponentially with the same rate, which has been calculated by semiclassical technics [31]. We found that the relaxation time $\tau$ above $T_{c}$ diverges like $\tau \sim K_{+}\left(T-T_{c}\right)^{-1 / 2}$, where $K_{+}$is known exactly. Approaching $T_{c}$ from below, the collapse time $t_{\text {coll }}$ diverges like $t_{\text {coll }} \sim K_{-}\left(T_{c}-T\right)^{-1 / 2}$, where again $K_{-}$has been computed analytically [32].

- In $d=2$, at $T_{c}$, the density obeys a dynamical scaling with a known scaling function $f$. The central density diverges like $\rho_{0}(t) \sim c_{1} \exp \left(c_{2} \sqrt{t}\right)$, where $c_{1}$ and $c_{2}$ are known universal constants [30, 34] (in an unbounded domain, the divergence is logarithmic instead of exponential 35] ). Below $T_{c}$, the collapse dynamics occurs after a finite time $t_{\text {coll }}$. At $t_{\text {coll }}$, a fraction $T / T_{c}$ of the total mass has condensed at $\mathbf{r}=\mathbf{0}$. Using the Virial theorem, we show that all the mass must collapse at $\mathbf{r}=\mathbf{0}$ in the post-collapse regime 35]. We mention these results in $d=2$, because the dynamics of the Bose-Einstein condensation in $d=3$ will share some qualitative analogy with this case.

We thus expect a surprising parallel between the collapse dynamics of a classical selfgravitating Brownian gas, which occurs when the kinetic pressure $P \sim T$ is not strong enough to counterbalance the gravitational attraction, and a bosonic gas, which forms a condensate at $\mathbf{k}=\mathbf{0}$, when the usual Bose-Einstein distribution is not able to accommodate for all the mass, even at zero chemical potential (see Section II.A). However, we note one 
fundamental difference between these two systems. For $t \rightarrow+\infty$, strictly below $T_{c}$ all the mass collapses at $\mathbf{r}=\mathbf{0}$ in the gravitational case, whereas the mass of the condensate is temperature-dependent in the case of the Bose-Einstein transition.

\section{Summary of results}

In Section II.A and II.B, we briefly review some basic results concerning the static properties of the Bose-Einstein condensation, and introduce a simpler model (SM), for which the static and dynamical properties can be analytically studied, and which will be equivalent in the dense region to the original Bose-Einstein model (BEM) described by Eqs. (2022). In section II.C, we show that Eqs. (20 22) maximize the rate of dissipation of bosonic free energy, and that the dynamical instability exactly coincides with the thermodynamical instability giving rise to the Bose-Einstein condensation below $T_{c}$.

In Section III, we address the collapse dynamics at and below $T_{c}$. In Section III.A, we compute the density scaling profile which is found to be independent of the temperature $T \leq T_{c}$, up to a temperature-dependent multiplicative factor. In Section III.B, we show that at $T=T_{c}$ the central density (at $\mathbf{k}=\mathbf{0}$ ) diverges exponentially with time, with a rate which can be calculated analytically. We compute the exponentially decaying corrections to the final Bose-Einstein distribution with $\mu=0$. In Section III.C, we show that for $T<T_{c}$,

the chemical potential vanishes in a finite time and that $\sqrt{2 T \mu(t)} \sim c(T)\left(t_{\text {coll }}-t\right)$, where $c(T)$ is computed analytically close to $T_{c}$. We show that $t_{c o l l}$ diverges logarithmically as $T$ approaches $T_{c}$ from below. In Section III.D, we address the specific collapse dynamics obtained strictly at $T=0$.

In Section IV, we study the post-collapse dynamics arising for $T<T_{c}$, and for times $t>t_{\text {coll }}$. We show that the condensate mass initially grows linearly, with a slope which can be calculated exactly near $T_{c}$. For large times, the mass of the condensate saturates exponentially fast to its equilibrium value, with a rate analytically known close to $T_{c}$.

In Section $\mathrm{V}$, we consider the relaxation time above $T_{c}$, which is computed in different limits. This relaxation time does not diverge at $T_{c}$, but the time after which this relaxation regime occurs (that we call the equilibration time) diverges logarithmically as the temperature approaches $T_{c}$ from above. 


\section{EQUILIBRIUM PROPERTIES AND A SIMPLER MODEL IN A BOX}

\section{A. Bose-Einstein condensation for a gas of free bosons}

Let us briefly repeat the few steps leading to the Bose-Einstein condensation. Considering free bosons, the equilibrium occupation number at momentum $\mathbf{k}$ is

$$
\rho_{B E}(\mathbf{k})=\frac{1}{\exp \left(\frac{\beta k^{2}}{2}+\mu\right)-1} .
$$

The total mass is fixed to unity, and the chemical potential $\mu$ is defined implicitly by the total mass constraint (for simplicity, we set the geometrical factor $S_{d}=1$, which amounts to fixing $M=S_{d}=1$ )

$$
M=\int_{0}^{\infty} \rho_{B E}(k) k^{d-1} d k=1
$$

which can be rewritten as the identity

$$
\beta^{d / 2}=\int_{0}^{\infty} \frac{k^{d-1}}{\exp \left(\frac{k^{2}}{2}+\mu\right)-1} d k .
$$

Below a certain temperature $T_{c}$, this distribution cannot include all the mass. The critical temperature corresponds to $\mu=0$. Specializing to the case of dimension $d=3$ which is the focus of this paper (there is no condensation for $d<3$ ), we find

$$
\beta_{c}=\left(\frac{\pi}{2}\right)^{1 / 3} \zeta^{2 / 3}\left(\frac{3}{2}\right), \quad \beta_{c} \approx 2.20494 \ldots
$$

where $\zeta(x)=\sum_{k=1}^{+\infty} k^{-x}$ is the Riemann $\zeta$-function. Below $T_{c}$, the momentum distribution is the Bose-Einstein distribution with $\mu=0$ plus a Dirac peak at $\mathbf{k}=\mathbf{0}$ containing the excess of mass $M_{0}$, with

$$
M_{0}=1-\left(\frac{\beta_{c}}{\beta}\right)^{3 / 2} \sim \frac{3}{2} \frac{T_{c}-T}{T_{c}}, \quad \frac{T-T_{c}}{T_{c}} \ll 1 .
$$

Above $T_{c}$, we find the following asymptotics which will be useful later:

$$
\mu \sim \frac{9}{8 \pi^{2}} T_{c}^{-3}\left(\frac{T-T_{c}}{T_{c}}\right)^{2}, \quad \frac{T-T_{c}}{T_{c}} \ll 1,
$$

and

$$
\mu \sim \frac{3}{2} \ln T, \quad T \rightarrow+\infty
$$




\section{B. A simpler model in a box}

We now introduce a simpler model (hereafter denoted SM), which will share the same properties as our original model, as far as the condensation dynamics properties are concerned. In the kinetic equation Eq. (20), we replace $(\rho+1)$ with $\rho$, which should be valid in the dense region around $\mathbf{k}=\mathbf{0}$, during the formation of the condensate. We obtain

$$
\frac{\partial \rho}{\partial t}=\nabla_{\mathbf{k}} \cdot\left[T \nabla_{\mathbf{k}} \rho+\rho^{2} \mathbf{k}\right]
$$

and the following equation for a radial solution

$$
\frac{\partial \rho}{\partial t}=T\left(\frac{\partial^{2} \rho}{\partial k^{2}}+\frac{d-1}{k} \frac{\partial \rho}{\partial k}\right)+d \rho^{2}+2 k \rho \frac{\partial \rho}{\partial k} .
$$

The integrated density satisfies

$$
\frac{\partial M}{\partial t}=T\left(\frac{\partial^{2} M}{\partial k^{2}}-\frac{d-1}{k} \frac{\partial M}{\partial k}\right)+\frac{1}{k^{d-2}}\left(\frac{\partial M}{\partial k}\right)^{2},
$$

which is even more similar to the dynamical equation Eq. (30) for a gas of self-gravitating Brownian particles than Eq. (22). The stationary solution can be easily calculated

$$
\rho(\mathbf{k})=\frac{1}{\frac{\beta k^{2}}{2}+\mu},
$$

which amounts to replacing the function $\exp x$ is the Bose-Einstein distribution by the function $(1+x)$. Note that the distribution (41) is the Lorentzian. Since this distribution $\rho(\mathbf{k})$ is not integrable up to infinity in $d \geq 2$, we introduce a momentum cut-off

$$
k \leq \Lambda
$$

As before, the total mass constraint reads

$$
M=\int_{0}^{\Lambda} \frac{k^{d-1}}{\frac{\beta k^{2}}{2}+\mu} d k=1 .
$$

From now on, we set $d=3$, as well as in the rest of the paper. Then, the integrated density can be explicitly calculated

$$
M(k)=2 T\left(k-\sqrt{2 \mu T} \arctan \left(\frac{k}{\sqrt{2 \mu T}}\right)\right) .
$$

The density profile Eq. (41) can accommodate for all the mass up to the temperature

$$
\beta_{c}=2 \Lambda
$$


Below $T_{c}$, a Dirac peak appears at $\mathbf{k}=\mathbf{0}$, containing the excess mass

$$
M_{0}=\frac{T_{c}-T}{T_{c}}
$$

Just above $T_{c}$, the chemical potential vanishes in a manner similar to the original BoseEinstein model (BEM),

$$
\mu \sim \frac{1}{2 \pi^{2}} T_{c}^{-3}\left(\frac{T-T_{c}}{T_{c}}\right)^{2}, \quad \frac{T-T_{c}}{T_{c}} \ll 1,
$$

whereas at high temperatures, the two models are qualitatively different, as the particles momentum cannot spread up to infinity in the SM. Hence, the chemical potential converges at high temperatures

$$
\mu \rightarrow \frac{\Lambda^{3}}{3}, \quad T \rightarrow+\infty
$$

\section{Some properties of the bosonic Fokker-Planck equation}

Equation (18) belongs to a general class of nonlinear Fokker-Planck (NFP) equations considered by Kaniadakis [17], Frank [36] and Chavanis [37]. These nonlinear Fokker-Planck equations arise when the coefficients of diffusion, mobility and friction depend explicitly on the distribution function. There are different ways to write these NFP equations. One convenient form for our present purposes is [37]:

$$
\frac{\partial \rho}{\partial t}=-\nabla \cdot \mathbf{J}_{*}=\nabla \cdot\left[\frac{1}{\xi}\left(T \nabla \rho+\frac{1}{C^{\prime \prime}(\rho)} \nabla \Phi\right)\right],
$$

where $C(\rho)$ is a convex function and $\xi>0$ can depend on $\mathbf{r}$ and $t$, e.g. on $\rho(\mathbf{r}, t)$. Here, $\Phi(\mathbf{r})$ is a fixed external potential but we can also consider the situation where $\Phi(\mathbf{r}, t)$ is generated by $\rho(\mathbf{r}, t)$ 37], like in the case of self-gravitating Brownian particles (see Section [B]). Taking $C(\rho)=\rho \ln \rho-\frac{1}{\kappa}(1+\kappa \rho) \ln (1+\kappa \rho)$, we recover the bosonic Fokker-Planck equation (18) for $\kappa=+1$, the fermionic Fokker-Planck equation for $\kappa=-1$ and the classical Fokker-Planck equation for $\kappa=0$ (for other values of $\kappa$, this describes intermediate "quon" statistics). For the simpler model Eq. (38), we have $C(\rho)=-\ln \rho$ describing logotropes [38]. Introducing a generalized free energy

$$
F=E-T S=\int \rho \Phi d \mathbf{r}+T \int C(\rho) d \mathbf{r}
$$

one can show that $F(t)$ is monotonically decreasing: $\dot{F}=-\int \xi C^{\prime \prime}(\rho) \mathbf{J}_{*}^{2} d \mathbf{r} \leq 0$. Therefore, if $F$ is bounded from below, the density $\rho(\mathbf{r}, t)$ will converge, for $t \rightarrow+\infty$, to the stationary 
solution $\rho_{e q}(\mathbf{r})$ such that $\mathbf{J}_{*}=\mathbf{0}$ (in that case $F$ is called a Lyapunov functional). The stationary solution of Eq. (49) is determined by

$$
\rho_{e q}(\mathbf{r})=\left(C^{\prime}\right)^{-1}[-\beta \Phi(\mathbf{r})-\alpha]
$$

where $\beta=1 / T$ and $\alpha$ is an integration constant. This stationary solution is a critical point of free energy Eq. (50) at fixed mass, i.e. it satisfies $\delta F+\alpha T \delta M=0$, where $\alpha T$ is a Lagrange multiplier. Moreover, it is shown in [37] that a stationary solution of Eq. (49) is linearly dynamically stable if, and only if, it is a minimum of $F$ at fixed mass. In fact, when $\Phi(\mathbf{r})$ is an external potential, the critical points of $F$ at fixed mass are necessarily minima since $\delta^{2} F=\frac{1}{2} T \int C^{\prime \prime}(\rho)(\delta \rho)^{2} d \mathbf{r} \geq 0$. Therefore, if a critical point of free energy exists it is the only minimum of $F$ and, consequently, $F$ is bounded from below: $F[\rho] \geq F\left[\rho_{e q}\right]$. In that case, the dynamical equation Eq. (49) will relax towards $\rho_{e q}(\mathbf{r})$ for $t \rightarrow+\infty$.

The NFP equation Eq. (49) can be justified in several different ways [37]. It can be obtained from the linear thermodynamics of Onsager by relating the current to the gradient of a "chemical potential" $\alpha(\mathbf{r}, t)=-\beta \Phi(\mathbf{r})-C^{\prime}(\rho)$ that is uniform at equilibrium (see Eq. (51) ), writing $\mathbf{J}_{*}=1 /\left[\beta \xi C^{\prime \prime}(\rho)\right] \nabla \alpha$. The current can also be expressed as the functional derivative of the free energy, writing $\mathbf{J}_{*}=-1 /\left[\xi C^{\prime \prime}(\rho)\right] \nabla(\delta F / \delta \rho)$. Alternatively, the NFP equation can be obtained by writing $\partial_{t} \rho=-\nabla \cdot \mathbf{J}$ and looking for the optimal current $\mathbf{J}_{*}$ which maximizes the rate of free energy dissipation $\dot{F}[\mathbf{J}]=\int\left(T C^{\prime \prime}(\rho) \nabla \rho+\nabla \Phi\right) \mathbf{J} d \mathbf{r}$

for a bounded function $E_{d}[\mathbf{J}]=\frac{1}{2} \int \xi C^{\prime \prime}(\rho) \mathbf{J}^{2} d \mathbf{r}$ preventing arbitrarily large values of the current $\mathbf{J}$. This is the variational version of the linear thermodynamics of Onsager. The optimal current, that is solution of $\delta \dot{F}+\delta E_{d}=0$, is the one appearing in Eq. (49) and it satisfies $\dot{F}\left[\mathbf{J}_{*}\right]=-2 E_{d}\left[\mathbf{J}_{*}\right] \leq 0$. It indeed leads to the most negative value of $\dot{F}$ (under constraints) since $\delta^{2}\left(\dot{F}+E_{d}\right)=\delta^{2} E_{d}=\frac{1}{2} \int \xi C^{\prime \prime}(\rho)(\delta \mathbf{J})^{2} d \mathbf{r} \geq 0$. These methods emphasize the (generalized) thermodynamical structure of the NFP equation.

\section{COLLAPSE DYNAMICS}

\section{A. General scaling solutions for $T \leq T_{c}$}

We now consider the collapse dynamics occurring when the system is suddenly quenched to $T_{c}$ or below $T_{c}$ from high temperature. It is clear that both considered models should 
behave in a similar manner near the dense region at $\mathbf{k}=\mathbf{0}$. Let us first consider the condensation dynamics of the SM. We write

$$
M(k, t)=2 T\left(k-k_{0} \arctan \left(\frac{k}{k_{0}}\right)\right)+F\left(k, k_{0}\right),
$$

where $k_{0}(t)$ is uniquely defined by the condition

$$
\rho(k=0, t)=\frac{2 T}{k_{0}^{2}}
$$

ensuring that

$$
F\left(k \rightarrow 0, k_{0}\right) \sim k^{5}
$$

The normalization condition reads

$$
M(k=\Lambda, t)=1=2 T\left(\Lambda-k_{0} \arctan \left(\frac{\Lambda}{k_{0}}\right)\right)+F\left(\Lambda, k_{0}\right) .
$$

We now look for a scaling solution of the form

$$
F\left(k, k_{0}\right)=k_{0}^{\alpha} f\left(\frac{k}{k_{0}}\right),
$$

and introduce the scaling variable

$$
x=\frac{k}{k_{0}}
$$

This leads to

$$
M^{\prime}(k, t)=k^{2} \rho(k, t)=\frac{2 T k^{2}}{k^{2}+k_{0}^{2}}+k_{0}^{\alpha-1} f^{\prime}\left(\frac{k}{k_{0}}\right)
$$

where, from now on, $M^{\prime}(k, t)$ will denote the derivative with respect to momentum. In analogy with the equilibrium density Eq. (41), we observe that $\mu(t)=\beta k_{0}^{2}(t) / 2$ can be seen as an effective chemical potential which is expected to vanish as time increases.

In $d=3$, the dynamical equation for $M(k, t)$ is

$$
\frac{\partial M}{\partial t}=T\left(M^{\prime \prime}-\frac{2}{k} M^{\prime}\right)+\frac{M^{\prime 2}}{k} .
$$

Inserting the scaling ansatz, we find

$$
\begin{aligned}
\frac{\partial M}{\partial t} & =k_{0}^{\alpha-2} T\left(f^{\prime \prime}+2 \frac{x^{2}-1}{x\left(x^{2}+1\right)} f^{\prime}\right)+k_{0}^{2 \alpha-3} \frac{f^{\prime 2}}{x} \\
& =-2 \dot{k}_{0} T\left(\arctan (x)-\frac{x}{x^{2}+1}\right)+\dot{k}_{0} k_{0}^{\alpha-1}\left(\alpha f-x f^{\prime}\right) .
\end{aligned}
$$

It is clear that for $\alpha=1$, all the terms of this equation scale the same way, if one chooses $\dot{k}_{0} \sim k_{0}^{-1}$, i.e. $k_{0}(t) \sim\left(t_{\text {coll }}-t\right)^{1 / 2}$. However, we were able to prove analytically that all 
the solutions of the resulting scaling equation are non physical, leading to a density going to a constant for $k \gg k_{0}$. In addition, when we wrote Eq. (52), we implicitly assumed that the second term of the right-hand side (RHS) is a correction to the first one, which implies $\alpha>1$. Hence, we now consider $\alpha>1$, and matching the leading terms of Eq. (60), we conclude that

$$
\dot{k}_{0}=-c k_{0}^{\alpha-2}
$$

and that the scaling function introduced above satisfies

$$
f^{\prime \prime}+2 \frac{x^{2}-1}{x\left(x^{2}+1\right)} f^{\prime}=2 c\left(\arctan (x)-\frac{x}{x^{2}+1}\right),
$$

which can be integrated once, leading to

$$
f^{\prime}(x)=\frac{2 c}{3} \frac{x}{\left(x^{2}+1\right)^{2}}\left[\left(x^{4}+6 x^{2}-3\right) \arctan (x)+3 x-2 x^{3}-4 x \ln \left(x^{2}+1\right)\right] .
$$

Note that $f^{\prime}(x) / x^{2}$ is exactly the scaling function for the density as can be seen from Eq. (58). We give below some asymptotic results for $f(x)$ which will prove useful later:

$$
\begin{aligned}
f(x) & =\frac{\pi c}{6} x^{2}-\frac{4 c}{3} x+2 \pi c \ln x+\mathcal{O}(1), \quad x \rightarrow+\infty, \\
& =\frac{2 c}{15} x^{5}-\frac{8 c}{45} x^{7}+\frac{227 c}{1260} x^{9}+\mathcal{O}\left(x^{11}\right), \quad x \rightarrow 0 .
\end{aligned}
$$

Let us now analyze the validity of the scaling regime. We ask that the neglected terms in Eq. (60) remain small in the range $k_{0} \ll k \ll \Lambda$. Using the large $x$ asymptotic for $f(x)$, we find that the non linear term in $f$ appearing in Eq. (60) can be neglected, if

$$
k_{0}^{2 \alpha-3} \frac{f^{\prime 2}}{x} \ll k_{0}^{\alpha-2} \Rightarrow k \ll k_{0}^{-(\alpha-2)},
$$

implying $\alpha \geq 2$. Similarly, the term arising from the time derivative of the residual density is negligible provided that

$$
\begin{aligned}
\dot{k}_{0} k_{0}^{\alpha-1}\left(\alpha f-x f^{\prime}\right) \ll k_{0}^{\alpha-2} & \Rightarrow \quad k \ll k_{0}^{\frac{3-\alpha}{2}} \quad(\alpha \neq 2), \\
& \Rightarrow \quad k \ll \Lambda \quad(\alpha=2),
\end{aligned}
$$

which implies that $\alpha \geq 3$ or $\alpha=2$. We will find in the next subsections that the admissible values $\alpha=3$ and $\alpha=2$ are in fact respectively associated to the collapse dynamics at $T_{c}$ and strictly below $T_{c}$.

We would like to emphasize that the present model provides an interesting example where one obtains a scaling solution which scales differently from what would have been obtained from a naive power counting, assuming that all terms in Eq. (60) scale the same way (which would lead to $\alpha=1)$. 


\section{B. Collapse at $T=T_{c}$}

At $T_{c}$, the constant appearing in the scaling function is denoted $c_{c}$. Expressing the conservation of mass, we find

$$
\begin{aligned}
1-2 T_{c}\left(\Lambda-k_{0} \arctan \left(\frac{\Lambda}{k_{0}}\right)\right) & =F\left(\Lambda, k_{0}\right) \sim k_{0}^{\alpha} f\left(\frac{\Lambda}{k_{0}}\right), \\
\frac{\pi}{2} \Lambda^{-1} k_{0} & \sim \frac{\pi c_{c}}{6} \Lambda^{2} k_{0}^{\alpha-2}+\mathcal{O}\left(k_{0}^{2}\right),
\end{aligned}
$$

which implies that

$$
\alpha=3, \quad c_{c} \sim \Lambda^{-3} \sim T_{c}^{3} .
$$

Inserting this value for $\alpha$ in Eq. (61), we find that $k_{0}(t)$ decays exponentially at $T_{c}$, with a rate $c_{c}$. To complete the computation of the scaling function, we need to determine the constant $c_{c}$ appearing as a prefactor in Eq. (63), and which also controls this exponential decay. Although the functional form of the scaling functions are identical for the SM and BEM, we will see that the constant $c_{c}$ will differs for both models, as this constant is not entirely determined by the dynamics in the region of high density, where the term $(\rho+1)$ can be safely replaced by $\rho$. To make this point clearer, we now look for a global solution for the correction term $F\left(k, k_{0}\right)$, valid for momentum much greater than $k_{0}$, and up to $k=\Lambda$ :

$$
F\left(k, k_{0}\right) \approx k_{0} \phi(k), \quad k_{0} \ll k \leq \Lambda
$$

Note that the large $x=\frac{k}{k_{0}}$ asymptotic of the scaling function should match the small $k$ behavior of $\phi(k)$ :

$$
k_{0} \phi(k) \sim_{k \rightarrow 0} k_{0}^{3} f\left(\frac{k}{k_{0}}\right) \sim_{k \gg k_{0}} \frac{\pi c_{c}}{6} k_{0} k^{2} .
$$

For the SM, expanding the integrated density up to leading order in $k_{0}$

$$
M(k, t)=2 T_{c}\left(k-\frac{\pi}{2} k_{0}\right)+k_{0} \phi(k)+\mathcal{O}\left(k_{0}^{2}\right),
$$

we immediately find that

$$
\phi(\Lambda)=\pi T_{c}
$$

On the other hand, substituting Eq. (174) in Eq. (40) we obtain to leading order in $k_{0}$ :

$$
\frac{\partial M}{\partial t}=k_{0} T_{c}\left(\phi^{\prime \prime}+\frac{2}{k} \phi^{\prime}\right)=\dot{k}_{0}\left(\phi(k)-\pi T_{c}\right)=-c_{c} k_{0}\left(\phi(k)-\pi T_{c}\right),
$$

so that $\phi(k)$ satisfies

$$
\phi^{\prime \prime}+\frac{2}{k} \phi^{\prime}-\beta_{c} c_{c}(\phi(\Lambda)-\phi)=0
$$


This equation admits the solution

$$
\phi(k)=\pi T_{c}\left(1-\frac{\sin \left(\sqrt{\beta_{c} c_{c}} k\right)}{\sqrt{\beta_{c} c_{c}} k}\right),
$$

and expressing the condition of Eq. (75), we determine $c_{c}$ and the full function $\phi(k)$

$$
c_{c}=4 \pi^{2} T_{c}^{3}, \quad \phi(k)=\pi T_{c}\left(1-\frac{\sin (\pi k / \Lambda)}{\pi k / \Lambda}\right) .
$$

It is straightforward to check that $\phi(k)$ obeys the small $k$ behavior of Eq. (73) , identical to the $k \gg k_{0}$ behavior of the scaling function $f$.

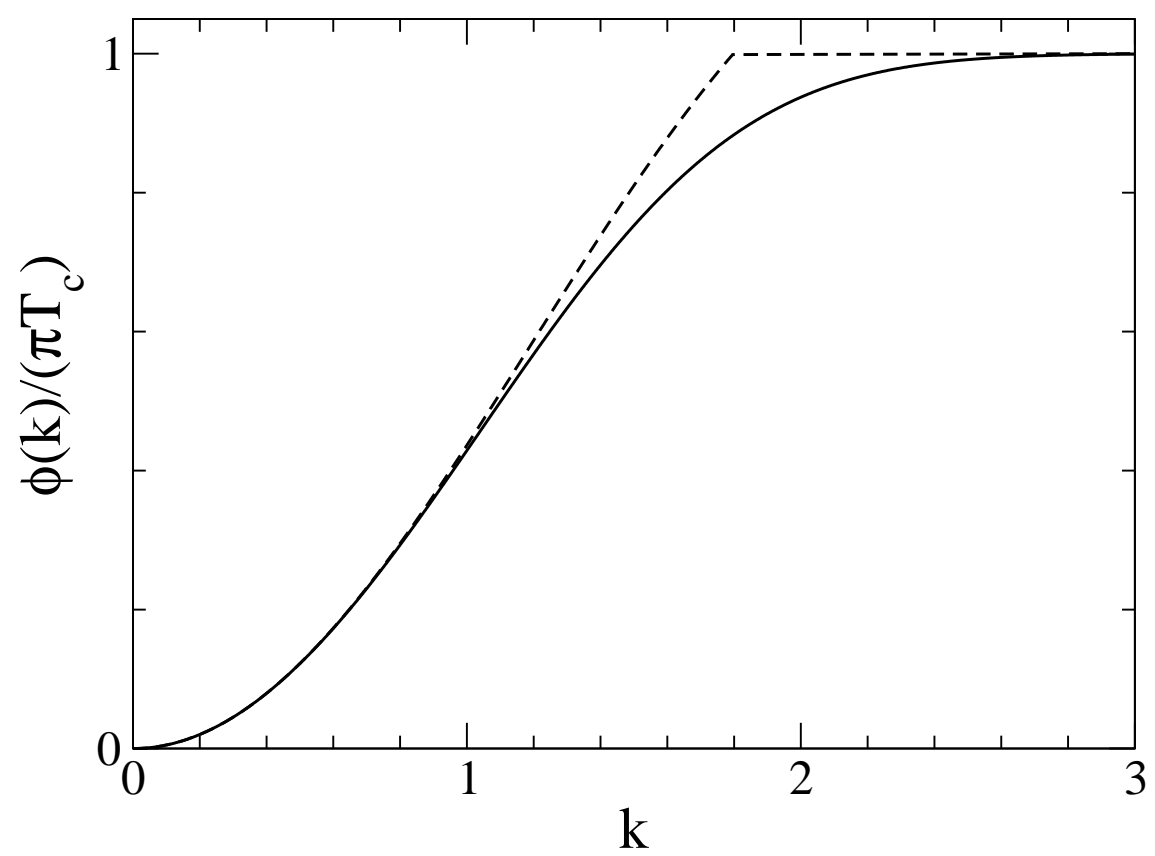

FIG. 1: We plot the analytical expressions of $\phi(k)$ (see Eq. (79), dashed line) for the simplified model compared to the numerical solution of Eq. (85) (full line). We have chosen the size of the confining box to be $\Lambda=1.79805 \ldots$ so that the small $k$ behavior of the two functions coincides.

Finally, the collapse at $T_{c}$ for the SM has been fully analyzed, and the central density (53) diverges as

$$
\rho(0, t)=2 T_{c} k_{0}^{-2}(t) \sim \exp \left(8 \pi^{2} T_{c}^{3} t\right)
$$

For infinite time, one converges to the expected equilibrium solution

$$
M(k, \infty)=2 T_{c} k, \quad \rho(k, \infty)=2 T_{c} k^{-2}
$$




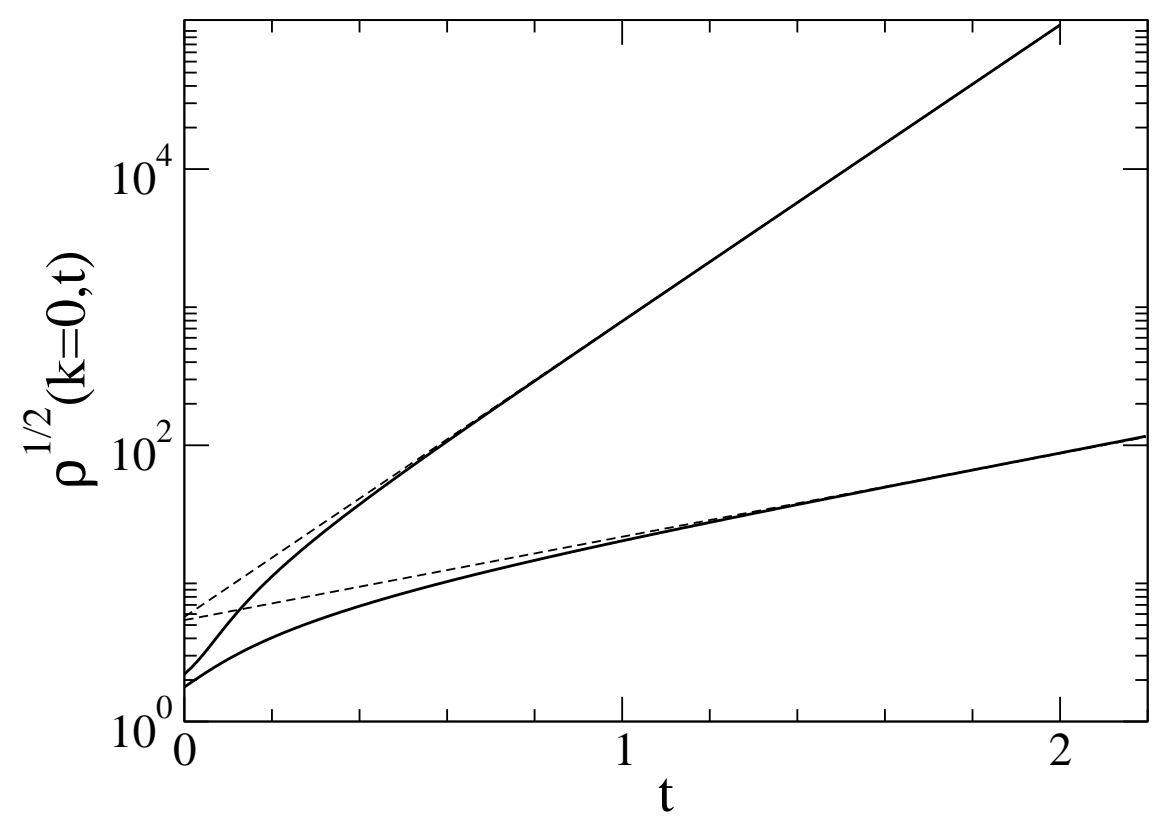

FIG. 2: At $T=T_{c}$, we plot $\rho^{1 / 2}(k=0, t) \sim k_{0}^{-1}(t) \sim \exp \left(c_{c} t\right)$ computed numerically by integrating the dynamical equation for $M(k, t)$ (see Eq. (59)), for the SM $(\Lambda=1$, top full line) and the BEM (bottom full line). In both cases, we find an exponential growth, with a rate in perfect agreement with our exact results for $c_{c}$ (the straight dashed lines in these semi-log plots have a slope equal to the theoretical values for $\left.c_{c}\right)$. For both models, we obtain an excellent fit of $\rho^{1 / 2}(k=0, t)$ to the functional form $\rho^{1 / 2}(k=0, t)=A \exp \left(c_{c} t\right)-B$, with $A \sim 5.7$ and $B \sim 0.5$ (fit not shown, but indistinguishable from data starting from $t \sim 0.2$ ).

Now, this approach can be repeated for the BEM, starting with a similar ansatz

$$
M(k, t)=\int_{0}^{k} \frac{k^{\prime 2}}{\exp \left(\beta_{c} \frac{k^{\prime 2}+k_{0}^{2}}{2}\right)-1} d k^{\prime}+k_{0} \phi(k),
$$

where $\mu(t)=\beta_{c} k_{0}^{2} / 2$ acts like a time-dependent chemical potential. For $k \gg k_{0}$, we find that

$$
\int_{0}^{k} \frac{k^{\prime 2}}{\exp \left(\beta \frac{k^{\prime 2}+k_{0}^{2}}{2}\right)-1} d k^{\prime}=\int_{0}^{k} \frac{k^{\prime 2}}{\exp \left(\beta \frac{k^{\prime 2}}{2}\right)-1} d k^{\prime}-\pi T k_{0}+\mathcal{O}\left(k_{0}^{2}\right) .
$$

Using the above result, we can insert the expression of $M(k, t)$ from Eq. (82) in the dynamical equation Eq. (59), and find up to the leading order in $k_{0}$

$$
\frac{\partial M}{\partial t}=k_{0} T_{c}\left[\phi^{\prime \prime}+\left(\frac{\beta_{c} k}{\tanh \left(\frac{\beta_{c} k^{2}}{4}\right)}-\frac{2}{k}\right) \phi^{\prime}\right]=\dot{k}_{0}\left(\phi(k)-\pi T_{c}\right)=-c_{c} k_{0}\left(\phi(k)-\pi T_{c}\right)
$$


or, after simplification,

$$
\phi^{\prime \prime}+\left(\frac{\beta_{c} k}{\tanh \left(\frac{\beta_{c} k^{2}}{4}\right)}-\frac{2}{k}\right) \phi^{\prime}-\beta_{c} c_{c}(\phi(\infty)-\phi)=0
$$

with

$$
\phi(\infty)=\pi T_{c}
$$

Now, $c_{c}$ is selected by imposing that $\phi(k)$ is an increasing function (being the integral of a density) and that one gets a fast decay of $\phi(\infty)-\phi(k) \sim \exp \left(-\beta k^{2} / 2\right)$. Solving this eigenvalue problem numerically, we find

$$
c_{c}=1.38452425 \ldots=C \pi^{2} T_{c}^{3}, \quad C=1.50380614 \ldots
$$

which leads to

$$
\rho(0, t)=2 T_{c} k_{0}^{-2}(t) \sim \exp \left(2 C \pi^{2} T_{c}^{3} t\right) .
$$

In Fig. 1, we have plotted the function $\phi(k)$ for the BEM, compared to its analytical counterpart for the SM. As expected, they significantly differ only for large momenta. In Fig. 2 , we numerically confirm the exponential growth of the density of particles at $\mathbf{k}=\mathbf{0}$ for both considered models, with the growth rate $c_{c}$ in perfect agreement with the present theoretical analysis. Finally, in Fig. 3, we illustrate the scaling behavior of the density profile.

\section{Collapse for $0<T<T_{c}$}

We now repeat the above procedure below $T_{c}$. For the SM model, expressing conservation of mass, we obtain

$$
\begin{aligned}
1-2 T\left(\Lambda-k_{0} \arctan \left(\frac{\Lambda}{k_{0}}\right)\right) & =F\left(\Lambda, k_{0}\right) \sim k_{0}^{\alpha} f\left(\frac{\Lambda}{k_{0}}\right), \\
\frac{T_{c}-T}{T_{c}} & \sim \frac{\pi c}{6} \Lambda^{2} k_{0}^{\alpha-2}+\mathcal{O}\left(k_{0}\right),
\end{aligned}
$$

which implies that

$$
\alpha=2, \quad c(T) \sim T_{c}\left(T_{c}-T\right)
$$

For this value of $\alpha$, Eq. (61) can be integrated, leading to

$$
k_{0}(t)=c(T)\left(t-t_{c o l l}\right)
$$




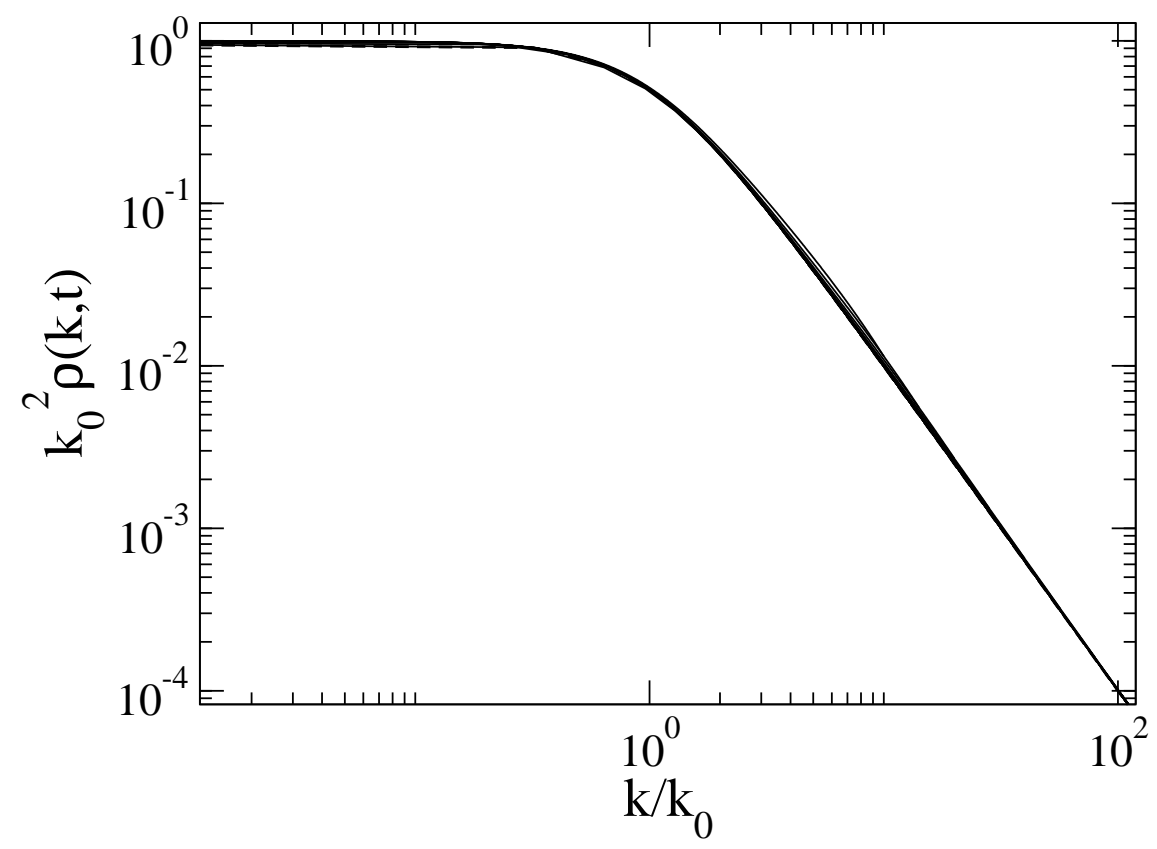

FIG. 3: At $T=T_{c}$, we plot $\left(2 T_{c}\right)^{-1} k_{0}^{2} \rho(k, t)$ as a function of $x=k / k_{0}$ for the BEM, for times for which the central density $\rho(0, t)=2 T_{c} k_{0}^{-2}$ is equal to $100 \times 2^{n}(n=1, \ldots, 14)$. We have also plotted the corresponding scaling function $1 /\left(1+x^{2}\right)$ (dashed line), which is perfectly superimposed with the data collapse. A similar plot for the SM would be indistinguishable, for the scale of momentum shown. Below $T_{c}$, a similar scaling arises for both models.

We thus find that, below $T_{c}$, a singularity should arise in a finite time, for which the central density diverges when $t$ goes to $t_{\text {coll }}$.

In order to obtain analytical results, we focus on the collapse dynamics just below $T_{c}$, so that $\frac{T-T_{c}}{T_{c}} \ll 1$, hence $F\left(k, k_{0}\right) \ll 1$ and $c \ll 1$. In this regime, non linear terms in $F$ can be safely neglected. For momenta $k \gg k_{0}$, we define a function $\phi(k)$ such that

$$
F\left(k, k_{0}\right) \approx\left(1+\frac{\lambda}{c} k_{0}\right) \phi(k), \quad k_{0} \ll k \leq \Lambda,
$$

resulting in the following expression for the integrated mass density

$$
M(k, t)=2 T\left(k-\frac{\pi}{2} k_{0}\right)+\left(1+\frac{\lambda}{c} k_{0}\right) \phi(k)+\mathcal{O}\left(k_{0}^{2}\right) .
$$

Taking $k=\Lambda$ and matching terms of order $\mathcal{O}\left(k_{0}^{0}\right)$ and $\mathcal{O}\left(k_{0}^{1}\right)$, we deduce that

$$
\phi(\Lambda)=\frac{T_{c}-T}{T_{c}}=\pi \frac{c}{\beta \lambda} .
$$


Inserting the expression of $M(k, t)$ in the dynamical equation Eq. (59), we obtain

$$
\frac{\partial M}{\partial t}=T\left(\phi^{\prime \prime}+\frac{2}{k} \phi^{\prime}\right)=\pi c T-\lambda \phi
$$

or

$$
\phi^{\prime \prime}+\frac{2}{k} \phi^{\prime}-\beta \lambda(\phi(\Lambda)-\phi)=0 .
$$

We recognize the very same equation for $\phi(k)$ as in the preceding section, so that the two functions are identical up to a multiplicative constant. Hence, for the SM, we obtain

$$
\lambda=c_{c}=4 \pi^{2} T_{c}^{3}, \quad c(T)=4 \pi T_{c}\left(T_{c}-T\right), \quad \phi(k)=\frac{T_{c}-T}{T_{c}}\left(1-\frac{\sin (\pi k / \Lambda)}{\pi k / \Lambda}\right),
$$

and a power law divergence of the central density

$$
\rho(0, t)=2 T k_{0}^{-2}(t) \sim \frac{1}{8 \pi^{2} T_{c}^{3}}\left(\frac{T_{c}-T}{T_{c}}\right)^{-2}\left(t_{\text {coll }}-t\right)^{-2} .
$$

For the SM, we can evaluate $\phi(k), c(T)$, and $\lambda(T)$ at the next order in $\delta=\frac{T_{c}-T}{T_{c}}$. We write

$$
\begin{aligned}
\phi(k) & =\delta \phi_{0}(k)+\delta^{2} \phi_{1}(k), \\
c(T) & =\delta c_{0}+\delta^{2} c_{1} \\
\lambda(T) & =\lambda_{0}+\delta \lambda_{1}
\end{aligned}
$$

where $\phi_{0}(k), c_{0}$ and $\lambda_{0}=c_{c}$ can be easily determined from Eq. (98). Inserting the above ansatz in the dynamical equation leads to a complicated linear equation for $\phi_{1}(k)$. We must impose the boundary conditions $\phi_{1}(0)=\phi_{1}^{\prime}(0)=0$ and $\phi_{1}(\Lambda)=0$, which fix the value of $c_{1}$ and $\lambda_{1}$. After some cumbersome but straightforward calculations, we obtain

$$
\begin{aligned}
\phi_{1}\left(x=\pi \frac{k}{\Lambda}\right)= & \frac{\sin x}{x}\left[\frac{c_{1}}{8 \pi T_{c}^{2}}\left(1+\frac{\pi-x}{\tan (x)}\right)+\frac{2 \pi \cos (x)}{3 x}\right. \\
& +\frac{3 \pi}{4 \tan (x)}\left(\int_{\pi}^{x} \frac{\cos (t)}{t} d t-\int_{3 \pi}^{3 x} \frac{\cos (t)}{t} d t\right) \\
& +\frac{\pi}{4}\left(3 \int_{3 x}^{+\infty} \frac{\sin (t)}{t} d t-\frac{11}{3} \int_{x}^{+\infty} \frac{\sin (t)}{t} d t\right) \\
& \left.+\frac{\pi(\cos (2 x)-1)}{6 x^{2} \sin (x)}\right],
\end{aligned}
$$

with

$$
\begin{aligned}
c_{1} & =\frac{2 \pi T_{c}^{2}}{3}\left(9 \ln (3)-4-9 \int_{\pi}^{3 \pi} \frac{\cos (t)}{t} d t\right) \\
& =C_{1} T_{c}^{2}, \quad C_{1}=13.51919467982 \ldots
\end{aligned}
$$


and

$$
\lambda_{1}=\pi C_{1} T_{c}^{3}
$$

Finally, we obtain

$$
\begin{aligned}
& c(T)=4 \pi T_{c}\left(T_{c}-T\right)+C_{1}\left(T_{c}-T\right)^{2}+\mathcal{O}\left(\left(T-T_{c}\right)^{3}\right), \\
& \lambda(T)=4 \pi T_{c}^{3}+\pi C_{1} T_{c}^{2}\left(T_{c}-T\right)+\mathcal{O}\left(T_{c}\left(T-T_{c}\right)^{2}\right),
\end{aligned}
$$

and note that the next correction to $c(T)$ and $\lambda(T)$ are both positive.

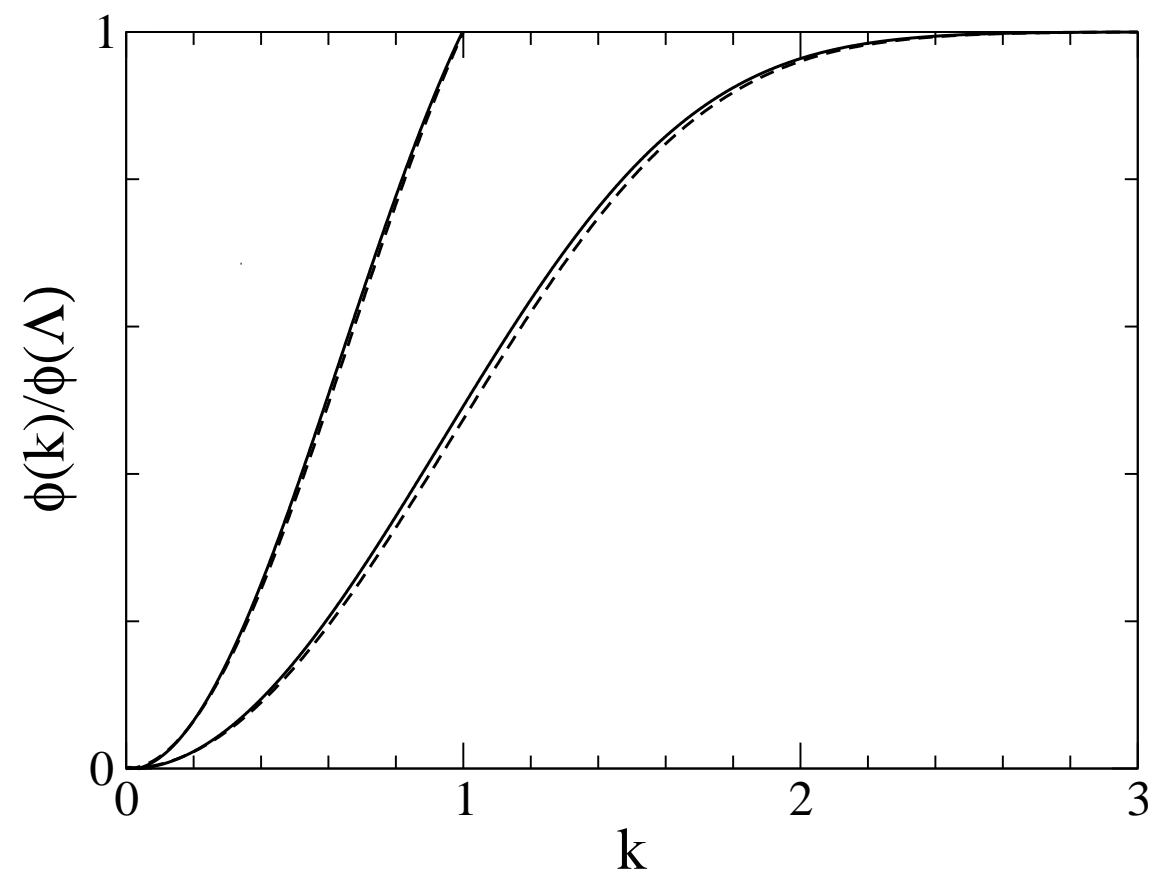

FIG. 4: For the simplified model, we plot $\phi(k)=M\left(k, t_{\text {coll }}\right)-2 T k$ obtained by numerically integrating the dynamical equation for $M(k, t)$ at $T=0.9 T_{c}$ (top full line). We have chosen $\Lambda=1$ so that $T_{c}=1 / 2$ and $\phi(\Lambda)=\frac{T_{c}-T}{T_{c}}=1 / 10$ (see Eq. (95)). It is in good agreement with our analytical expression of Eq. (98) (top dashed line), which is strictly valid only very close to $T_{c}$. We also plot the numerical (bottom full line) and theoretical expression of $\phi(k)$ (bottom dashed line), for the Bose-Einstein model $\left(\phi(\Lambda=\infty)=1-27 / 10^{3 / 2}=0.1462 \ldots \approx \frac{3}{2} \frac{T_{c}-T}{T_{c}}\right)$.

Again, this approach can be repeated for the more realistic BEM, by writing

$$
M(k, t)=\int_{0}^{k} \frac{k^{\prime 2}}{\exp \left(\beta \frac{k^{\prime 2}+k_{0}^{2}}{2}\right)-1} d k^{\prime}+\left(1+\frac{\lambda}{c} k_{0}\right) \phi(k) .
$$


At the leading order in $k_{0}$, the dynamical equation Eq. (59) leads to

$$
\frac{\partial M}{\partial t}=T\left[\phi^{\prime \prime}+\left(\frac{\beta k}{\tanh \left(\frac{\beta k^{2}}{4}\right)}-\frac{2}{k}\right) \phi^{\prime}\right]=\pi c T-\lambda \phi
$$

or more simply to

$$
\phi^{\prime \prime}+\left(\frac{\beta k}{\tanh \left(\frac{\beta k^{2}}{4}\right)}-\frac{2}{k}\right) \phi^{\prime}-\beta \lambda(\phi(\infty)-\phi)=0
$$

which is again the same equation as the one found in the previous section, which determines the function $\phi(k)$ up to a multiplicative constant. In order to compute this constant, we have to match the terms of order $\mathcal{O}\left(k_{0}^{0}\right)$ and $\mathcal{O}\left(k_{0}^{1}\right)$ for $k \rightarrow+\infty$ in Eq. (109), which implies that

$$
\begin{aligned}
\phi(\infty) & =1-\left(\frac{\beta_{c}}{\beta}\right)^{3 / 2} \approx \frac{3}{2} \frac{T_{c}-T}{T_{c}} \\
& =\pi \frac{c}{\beta \lambda}
\end{aligned}
$$

Finally, we obtain

$$
\lambda=c_{c}=C \pi^{2} T_{c}^{3}, \quad c(T)=C^{\prime} \pi T_{c}\left(T_{c}-T\right), \quad C^{\prime}=\frac{3 C}{2}=2.2557092066 \ldots
$$

and

$$
\rho(0, t)=2 T k_{0}^{-2}(t) \sim \frac{2}{C^{\prime 2} \pi^{2} T_{c}^{3}}\left(\frac{T_{c}-T}{T_{c}}\right)^{-2}\left(t_{c o l l}-t\right)^{-2} .
$$

Note that at $t=t_{\text {coll }}$, we find that the density and the integrated density are

$$
\rho\left(k, t_{\text {coll }}\right)=\frac{2 T}{k^{2}}+\frac{\phi^{\prime}(k)}{k^{2}}, \quad M\left(k, t_{\text {coll }}\right)=2 T k+\phi(k),
$$

for the simplified model, and

$$
\rho\left(k, t_{\text {coll }}\right)=\frac{1}{\exp \left(\beta \frac{k^{2}}{2}\right)-1}+\frac{\phi^{\prime}(k)}{k^{2}}, \quad M\left(k, t_{\text {coll }}\right)=\int_{0}^{k} \frac{k^{\prime 2}}{\exp \left(\beta \frac{k^{\prime 2}}{2}\right)-1} d k^{\prime}+\phi(k),
$$

for the more realistic Bose-Einstein model. In addition to the equilibrium density, we obtain in both models a singular contribution near $k=0$

$$
\frac{\phi^{\prime}(k)}{k^{2}} \sim \frac{\pi}{3 k} c(T), \quad k \rightarrow 0
$$




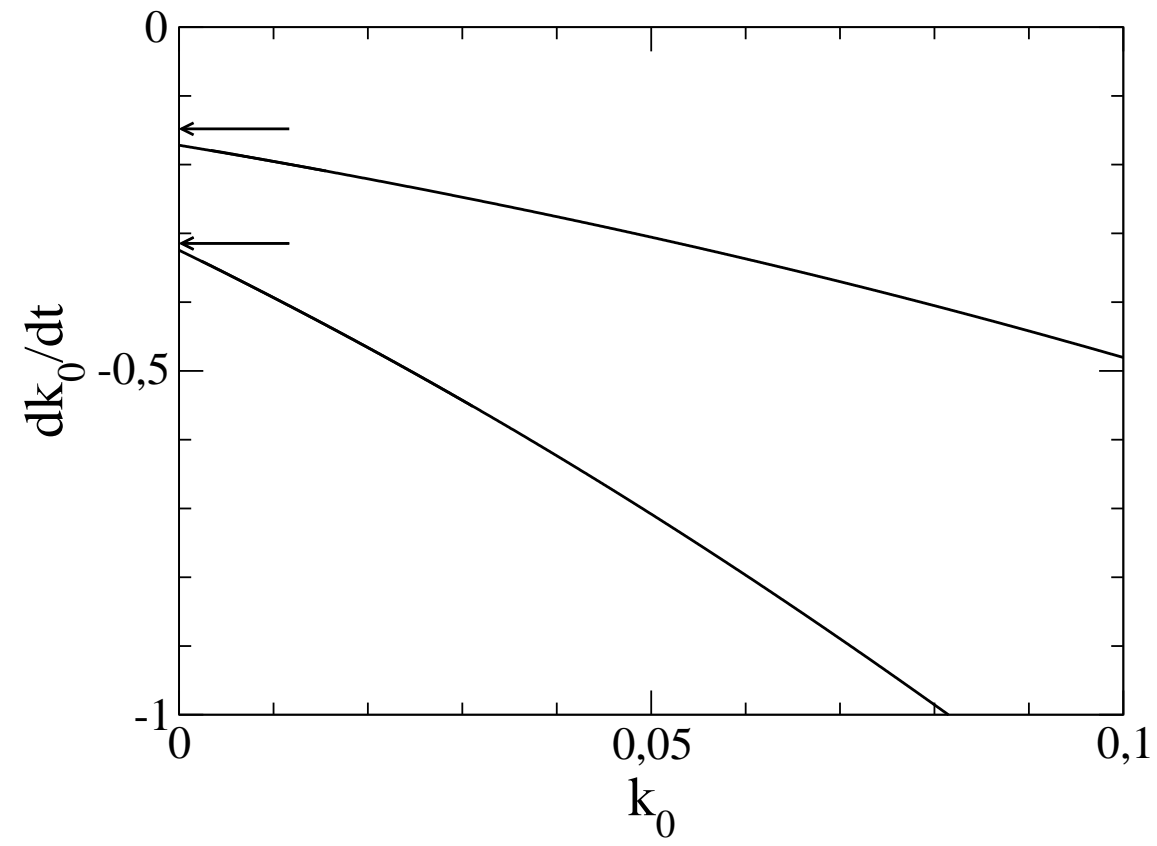

FIG. 5: For the simplified model $(\Lambda=1$, bottom line) and the Bose-Einstein model (top line), we plot $\dot{k}_{0}(t) \rightarrow-c(T)$ as a function of $k_{0}(t)$ as obtained by integrating the dynamical equation for $M(k, t)$ at $T=0.9 T_{c}$. The theoretical slopes $-c(T)$ evaluated at the first order in $T_{c}-T$ are indicated by an arrow. We find that the numerical value of $c(T)$ are slightly bigger than our first order calculation, consistent with the fact that the next correction is analytically found to be positive for the SM (see Eq. (107)). For small $k_{0}(t)$ (i.e. close to $t_{\text {coll }}$ ), we find $\dot{k}_{0}(t) \approx$ $-c(T)-A c_{c} k_{0}(t)$, with $A \approx 1.35$ for the SM, and $A \approx 1.65$ for the BEM.

However, we do not observe the appearance of a Dirac peak at $\mathbf{k}=\mathbf{0}$, implying that the evolution of the system is not finished yet. Hence, we expect that after $t_{c o l l}$, the mass included in $\phi(k)$ will be swallowed at $k=0$, finally giving rise to the condensate.

Before addressing this issue, we give an estimate of $t_{\text {coll }}$, near $T_{c}$. If one is very close to $T_{c}$, the density first grows exponentially like at $T_{c}$, before crossing over to the behavior of Eq. (115), when the time is of order $t_{\text {coll }}$. Matching the two regimes, we obtain

$$
\rho\left(k=0, t \sim t_{\text {coll }}\right) \sim \exp \left(2 c_{c} t_{\text {coll }}\right) \sim\left(\frac{T_{c}-T}{T_{c}}\right)^{-2} t_{\text {coll }}^{-2}
$$

leading to the rough estimate

$$
t_{\text {coll }} \sim T_{c}^{-3} \ln \left(\frac{T_{c}}{T_{c}-T}\right)
$$

As expected, $t_{\text {coll }}$ diverges as the temperature approaches $T_{c}$. This analysis suggests a global 
form for $\dot{k}_{0}(t)$, valid for all temperature

$$
\dot{k}_{0}=-c(T) h\left(\frac{c_{c}}{c(T)} k_{0}\right), \quad h(0)=1, \quad h(x) \sim_{\infty} x,
$$

where $c(T) \sim T_{c}\left(T_{c}-T\right)$ for both models. If we assume the simple form $h(x)=1+x$ (numerically we find $h(x)=1+A x$, for small $x$; see Fig. 5 ), which is compatible with the known asymptotics of $f(x)$ in Eq. (121), we obtain

$$
\dot{k}_{0}=-c_{c} k_{0}-c(T)
$$

This equation has a global solution

$$
k_{0}(t)=\frac{c(T)}{c_{c}}\left(\exp \left[c_{c}\left(t_{c o l l}-t\right)\right]-1\right) .
$$

For times $t$ close to $t_{\text {coll }}$, this expression leads to the asymptotic scaling of Eq. (92), whereas it also reproduces the early exponential decay with rate $c_{c}$, which is expected for $T$ close to $T_{c}$. Imposing that at $t=0, k_{0}$ should be of order of a typical momentum of the initial condition (for instance $k_{0} \sim \Lambda$ for the SM), we recover exactly the estimate of $t_{\text {coll }}$ obtained in Eq. (120).

In Fig. 4, we plot the numerical estimate of $\phi(k)$ obtained by numerically integrating Eq. (59) at $T=0.9 T_{c}$, and compare it to our theoretical results, which are, in principle, only valid very close to $T_{c}$. In Fig. 5 , we plot $\dot{k}_{0}(t) \rightarrow-c(T)$ obtained by numerically integrating Eq. (59) and find a fair agreement with our theoretical estimates for $c(T)$, which are strictly valid only close to $T_{c}$.

\section{Collapse at $T=0$}

For completeness, we now consider the collapse dynamics at $T=0$. Since we are interested in the density scaling function, we address this question within the simplified model. We consider the dynamical equation for the density rather than for the integrated density

$$
\frac{\partial \rho}{\partial t}=3 \rho^{2}+2 k \rho \rho^{\prime}
$$

Inserting a scaling ansatz of the form

$$
\rho(k, t)=\rho_{0} g\left(\frac{k}{k_{0}}\right),
$$


in the dynamical equation Eq. (124), we obtain

$$
\dot{\rho}_{0} g-\rho_{0} \frac{\dot{k}_{0}}{k_{0}} x g^{\prime}=\rho_{0}^{2}\left(3 g^{2}+2 x g g^{\prime}\right) .
$$

We introduce the parameter $\alpha$ defined by

$$
\frac{\dot{\rho}_{0}}{\rho_{0}}=-\alpha \frac{\dot{k}_{0}}{k_{0}}=\alpha \rho_{0} .
$$

Then, the equation for the scaling function becomes

$$
\frac{1-2 g}{g(\alpha-3 g)} g^{\prime}=-\frac{1}{x}
$$

which can be exactly solved, leading to the following implicit equation for $g(x)$

$$
g(x)\left(\frac{\alpha}{3}-g(x)\right)^{2 \alpha / 3-1}=C x^{-\alpha}
$$

where $C$ is a constant depending on the initial conditions, as was already noticed within the study of the gravitational collapse at $T=0$ [29, 30, 31]. Now, for small $x=k / k_{0}, g(x)$ must be an analytic function, with a small $x$ expansion of the form $g(x)=g(0)+g^{\prime \prime}(0) x^{2} / 2+\ldots$. Matching the small $x$ behavior of the RHS and LHS of Eq. (129), we obtain

$$
\alpha=\frac{6}{7}, \quad g(0)=\frac{2}{7}
$$

and

$$
g(x)\left(\frac{2}{7}-g(x)\right)^{-3 / 7}=C x^{-6 / 7} .
$$

We also obtain the exact expression for the central density, and the time-dependent width of the dense core $k_{0}(t)$

$$
\rho(k=0, t)=\frac{2}{7} \rho_{0}(t)=\frac{1}{3}\left(t_{\text {coll }}-t\right)^{-1}, \quad k_{0}(t)=\left(t_{\text {coll }}-t\right)^{7 / 6} .
$$

Finally, at $t=t_{\text {coll }}$, the small $k$ behavior of the density becomes universal

$$
M\left(k, t_{\text {coll }}\right) \sim k^{15 / 7}, \quad \rho\left(k, t_{\text {coll }}\right) \sim k^{-6 / 7}
$$

in contrast with the behavior obtained for $0<T \leq T_{c}$, where we found $\rho\left(k, t_{\text {coll }}\right) \sim 2 T k^{-2}$. 
IV. POST-COLLAPSE DYNAMICS FOR $0<T<T_{c}$

As mentioned in Section III.C, the density profile at $t=t_{\text {coll }}$ is not yet equal to the equilibrium profile which presents a Dirac peak at $\mathbf{k}=\mathbf{0}$. This means that after $t_{\text {coll }}$, the density profile should continue to relax to

$$
\rho(\mathbf{k})=\frac{T_{c}-T}{T_{c}} \delta(\mathbf{k})+2 T k^{-2}
$$

for the simplified model, and

$$
\rho(\mathbf{k})=\left[1-\left(\frac{\beta_{c}}{\beta}\right)^{3 / 2}\right] \delta(\mathbf{k})+\frac{1}{\exp \left(\beta \frac{k^{2}}{2}\right)-1},
$$

for the Bose-Einstein model. In order to obtain analytical results in this post-collapse stage, we again consider the case of a temperature just below $T_{c}$, such that

$$
\frac{T_{c}-T}{T_{c}} \ll 1
$$

In this regime, and for the SM, the following ansatz is an exact solution of the dynamical equation Eq. (59)

$$
M(k, t)=M_{0}(t)+2 T k+\left(1-\frac{M_{0}(t)}{\phi(\Lambda)}\right) \phi(k),
$$

where $M_{0}(t)$ is the time-dependent mass in the condensate, and where $\phi(k)$ has been calculated in the collapse regime in the previous section. Inserting this ansatz in the kinetic equation, we obtain

$$
\frac{\partial M}{\partial t}=\dot{M}_{0}\left(1-\frac{\phi(k)}{\phi(\Lambda)}\right)=T\left(1-\frac{M_{0}(t)}{\phi(\Lambda)}\right)\left(\phi^{\prime \prime}+\frac{2}{k} \phi^{\prime}\right),
$$

where we have neglected quadratic terms in $\phi(k)$, which is justified near $T_{c}$. For this equation to be compatible with Eq. (97), i.e. the defining equation for $\phi(k)$, we must have

$$
\dot{M}_{0}=c_{c}\left(\phi(\Lambda)-M_{0}(t)\right)
$$

which can be easily solved, leading to the full time dependence of the condensate mass $M_{0}(t)$

$$
M_{0}(t)=\frac{T_{c}-T}{T_{c}}\left(1-\exp \left[-4 \pi^{2} T_{c}^{3}\left(t-t_{c o l l}\right)\right]\right) .
$$

For the Bose-Einstein model, we proceed in a similar manner, and find for $T$ close to $T_{c}$

$$
\begin{aligned}
M_{0}(t) & =\left[1-\left(\frac{\beta_{c}}{\beta}\right)^{3 / 2}\right]\left(1-\exp \left[-C \pi^{2} T_{c}^{3}\left(t-t_{\text {coll }}\right)\right]\right), \\
& \approx \frac{3}{2} \frac{T_{c}-T}{T_{c}}\left(1-\exp \left[-C \pi^{2} T_{c}^{3}\left(t-t_{\text {coll }}\right)\right]\right) .
\end{aligned}
$$


For both models, the condensate mass initially grows linearly with time

$$
M_{0}(t)=\pi T_{c} c(T)\left(t-t_{\text {coll }}\right) \sim T_{c}^{2}\left(T_{c}-T\right)\left(t-t_{\text {coll }}\right), \quad t-t_{\text {coll }} \ll T_{c}^{-3},
$$

whereas it saturates exponentially fast to its equilibrium value, with a rate $c_{c}$ equal to the relaxation rate found at $T_{c}$ (see Section III.B and the next section), since one can write

$$
1-\frac{M_{0}(t)}{M_{0}(\infty)}=\exp \left[-c_{c}\left(t-t_{c o l l}\right)\right]
$$

\section{RELAXATION TIME FOR $T>T_{c}$}

Finally, in this section, we address the problem of determining the relaxation rate to the equilibrium solution above $T_{c}$. Writing

$$
M(k, t)=\int_{0}^{k} \frac{k^{\prime 2}}{\exp \left(\frac{\beta k^{\prime 2}}{2}+\mu\right)-1} d k^{\prime}+\epsilon(k) \exp (-t / \tau)
$$

and neglecting non linear terms in $\epsilon(k)$, we find that

$$
\epsilon(k)=h(\sqrt{\beta} k)
$$

where $h(x)$ satisfies

$$
h^{\prime \prime}+\left(\frac{x}{\tanh \left(\frac{x^{2}}{4}+\frac{\mu}{2}\right)}-\frac{2}{x}\right) h^{\prime}+\tau^{-1} h=0 .
$$

For $T \gg T_{c}$ (and hence $\mu \gg 1$ ), we can replace tanh by unity and the solution can be exactly written in term of the Kummer confluent hypergeometric function $K$

$$
h(x)=x^{3} K\left(\frac{3 \tau+1}{2 \tau}, \frac{5}{2},-\frac{x^{2}}{2}\right) .
$$

Imposing a fast decaying solution, we find

$$
h(x)=x^{3} \exp \left(-\frac{x^{2}}{2}\right), \quad \tau(T \rightarrow+\infty)=\frac{1}{2} .
$$

For the simplified model, the limit of high temperature is not physically interesting since, due to the finite box, the particles cannot spread up to large momenta.

For $0<T-T_{c} \ll T_{c}$ (and hence $\mu \ll 1$ ), the eigenequation Eq. (147) coincide with the defining equation for the function $\phi(k)$ introduced in Section III.B. Hence, $\tau\left(T \rightarrow T_{c}\right)=c_{c}^{-1}$, using the respective values of $c_{c}$ for the two models considered. 
It seems paradoxical that the relaxation time does not diverge at $T_{c}$, contrary to what is expected for a continuous phase transition. However, $\tau$ is not the equilibration time, which is the typical time $\tau_{e q}$ needed to reach the equilibrium solution. This time can be evaluated by considering that close but above $T_{c}$, the chemical potential initially decreases as $\beta k_{0}(t)^{2} / 2$ does at $T_{c}$, and reaches the order of magnitude of its equilibrium value after a time $\tau_{e q}$. Thus, we write

$$
k_{0}\left(\tau_{e q}, T_{c}\right) \sim \exp \left(-c_{c} \tau_{e q}\right) \sim k_{0}(T)=\sqrt{2 \mu T}
$$

Using the expressions for $\mu$ near $T_{c}$ given in Eq. (36) for the BEM and in Eq. (47) for the SM, we obtain

$$
\tau_{e q} \sim-T_{c}^{-3} \ln \mu \sim T_{c}^{-3} \ln \left(\frac{T_{c}}{T-T_{c}}\right)
$$

for both models. Note the similarity of this estimate with the expression of $t_{\text {coll }}$ near $T_{c}$, given in Eq. (120). The exponential relaxation controlled by $\tau$ only starts after a time of order $\tau_{e q} \gg \tau$.

\section{COMPARISON WITH OTHER WORKS}

In this section, we give a short review of classical works concerning the dynamics of the Bose-Einstein condensation to show how our results compare with these studies. The dynamics of the Bose-Einstein condensation has been described by two apparently different kinetic theories. The first kinetic theory is based on a quantum version of the Boltzmann equation for the one-particle distribution function $\rho(\mathbf{k}, t)$. This is a semi-classical approach which introduces corrections for quantum statistics into the ordinary Boltzmann collision term [10, 11, 12]. Another description is provided by the time-dependent Gross-Pitaevskii equation for the condensate wave function $\psi(\mathbf{x}, t)$ (order parameter for the Bose condensate) in a spatially homogeneous medium [39]. This equation does not take into account quantum fluctuations, or thermal or irreversible effects, but is valid when a large number of particles have condensed.

One of the first solution of these kinetic equations is due to Levich \& Yakhot 40] who consider a gas of bosons without interaction (i.e. neglecting collisions) in contact with a thermal bath of fermions. They provide an analytical solution of the corresponding quantum Boltzmann equation and find that the Bose condensate forms in an infinite time. Since 
this result is inconsistent with observations, Stoof [41] argues that the quantum Boltzmann equation is not valid to describe the condensate. He considers an isolated gas of bosons in interaction (i.e. with interatomic collisions) and argues that the dynamics of the collapse follows three steps: (i) an incoherent evolution described by the quantum Boltzmann equation (ii) a coherent evolution triggering a phase transition (instability) leading to the Bose condensate (iii) a thermalization between the condensate and the non condensed atoms interpreted as quasi-particles in the sense of Bogoliubov. Stoof focuses on the coherent evolution. Using a functional formulation of the Keldysh theory, he derives a time dependent Landau-Ginzburg equation for the order parameter $\langle\psi(\mathbf{x}, t)\rangle$ of the phase transition. He also shows that the transition temperature for interacting bosons is larger than for an ideal gas and that the nucleation of the condensation is short, contrary to the result of [40], except for temperatures close to the critical temperature $T_{c}$. In a more recent paper, Stoof [42] derives a Fokker-Planck equation for the probability distribution of the order parameter $\langle\psi(\mathbf{x}, t)\rangle$ which gives a unified description of both the incoherent (kinetic Boltzmann) and coherent (Gross-Pitaevskii) stages of Bose-Einstein condensation. A similar program of unification of the two theories is carried out by Gardiner \& Zoller [43]. Using a projection of the density operator $\rho$, they derive a quantum kinetic master equation (QKME) for bosonic atoms and recover, in particular limits, the quantum Boltzmann equation and the Gross-Pitaevskii equation.

Semikoz \& Tkachev [13] and Lacaze et al. [14] numerically solve the quantum Boltzmann equation and find the formation of a condensate in finite time and the growth of this condensate in a post-collapse regime. This is quite different from the results of Levich \& Yakhot [40] which are valid when the system is coupled to a bath of fermions. Therefore, the original bosonic Boltzmann kinetic equation (without fermion bath approximation) can account for a finite time formation of the condensate. More recently, using an analogy with optical turbulence, Connaughton \& Pomeau [44] obtain a kinetic equation for the spectral particle density $\rho(\mathbf{k}, t)$ directly from the Gross-Pitaevskii equation for the wave function $\psi(\mathbf{x}, t)$ when the non-linear term is considered as a perturbation ( $\mathbf{k}$ is the conjugate of $\mathbf{x}$ in the Fourier analysis). They show that this kinetic equation has the same form as the quantum Boltzmann equation.

Several authors [13, 14, 45, 46] have investigated self-similar solutions of the quantum Boltzmann equation in the form $\rho(\epsilon, t)=\rho_{0}(t) f\left(\epsilon / \epsilon_{0}(t)\right)$, where $\epsilon=\frac{k^{2}}{2 m}$. In particular, 
Semikoz \& Tkachev [13] find a pre-collapse regime generating, in a finite time $t_{\text {coll }}$, a distribution function of energies scaling as $f(\epsilon) \sim \epsilon^{-\alpha}$ with $\alpha=1.24$. This profile is slightly steeper than the Zakharov profile $\epsilon^{-7 / 6}$ providing an exact static solution of the quantum Boltzmann equation corresponding to a constant mass flux $J$ in momentum space toward the condensate. The central density increases as $\rho_{0}(t) \sim\left(t_{\text {coll }}-t\right)^{-\alpha / 2(\alpha-1)} \sim\left(t_{\text {coll }}-t\right)^{-2.6}$ and becomes infinite at $t=t_{\text {coll }}$. The typical core energy $\epsilon_{0}(t) \sim\left(t_{\text {coll }}-t\right)^{1 / 2(\alpha-1)} \sim\left(t_{\text {coll }}-t\right)^{2.1}$ goes to zero at $t=t_{\text {coll }}$. Semikoz \& Tkachev [13] also investigate the post-collapse regime. They find that the energy distribution passes from $\epsilon^{-\alpha}$ to $\epsilon^{-1}$ after the singularity and that the mass of the condensate grows like $n_{0}(t)=\left(t-t_{\text {coll }}\right)^{(3-2 \alpha) / 4(\alpha-1)} \sim\left(t-t_{\text {coll }}\right)^{0.54}$ just after

collapse. Lacaze et al. 14] also investigate self-similar solutions and obtain similar results with an exponent $\alpha=1.234$.

Our approach is physically different since we consider a gas of non-interacting bosons in contact with a heat bath establishing a Bose-Einstein distribution (canonical description) while the previous authors consider an isolated system of bosons in interaction (microcanonical description). However, although the dynamics differs in the details (as expected), the phenomenology of the collapse is almost the same and the simplified form of our kinetic equation (18) allows for a complete analytical solution of the problem (pre and post collapse) which is not possible for the quantum Boltzmann equation [13, 14, 45, 46]. This is clearly an interest of our model.

\section{CONCLUSION}

In the present paper, we have considered the condensation in $\mathbf{k}$-space of a homogeneous gas of non-interacting bosons in a thermal bath fixing the temperature $T$. In statistical mechanics, this corresponds to a canonical description. We have pointed out the striking analogy between the dynamical equation describing the Bose-Einstein condensation in the canonical ensemble and the one describing the evolution of a self-gravitating gas of Brownian particles (or the chemotaxis of bacterial populations).

In analogy to the case of a classical gas, we have constructed the dynamical Fokker-Planck equation for the momentum distribution. For $T<T_{c}$, the system experiences a finite time singularity, i.e. the density at $\mathbf{k}=\mathbf{0}$ becomes infinite in a finite time $t_{\text {coll }} \sim T_{c}^{-3} \ln \left(\frac{T_{c}}{T-T_{c}}\right)$. However, the "singularity contains no mass" and the condensate is formed during the post- 
collapse regime for $t>t_{\text {coll }}$. This dynamical scenario leads, for $t \rightarrow+\infty$, to a Dirac peak in addition to the Bose-Einstein distribution with zero chemical potential, which is entirely consistent with the distribution predicted by Einstein at equilibrium [9]. Our canonical description permits a complete analytical treatment for the different stages of the process, contrary to the microcanonical approach starting from the semi-classical Boltzmann equation [13, 14], where one has to mostly rely on numerical simulations. Although qualitatively similar (existence of a finite time pre-collapse regime for $t<t_{\text {coll }}$, scaling behavior of the density, formation of the condensate in the post-collapse regime...), we note that our results differ quantitatively from the ones obtained within the microcanonical approach. For instance, $k_{0}(t)=c(T)\left(t_{\text {coll }}-t\right)$ in our canonical model, whereas $k_{0}(t) \sim\left(t_{\text {coll }}-t\right)^{\gamma}$ in the microcanonical ensemble, where, numerically, one finds $\gamma \approx 1.07$ (the difference is even more apparent on other quantities) [14]. This should not be surprising, as the same phenomenon arises in the study of the collapse dynamics of a self-gravitating gas, which is different in the canonical and microcanonical ensembles, though qualitatively similar in many respects [29].

In this paper, we have assumed that the Bose gas is homogeneous. The inhomogeneous situation could be studied semiclassically by introducing a mean-field advective term in the kinetic equation such that

$$
\frac{\partial \rho}{\partial t}+\mathbf{k} \cdot \frac{\partial \rho}{\partial \mathbf{r}}-\nabla U(\mathbf{r}) \cdot \frac{\partial \rho}{\partial \mathbf{k}}=Q(\rho)
$$

where $Q(\rho)$ is either the bosonic Fokker-Planck operator of Eq. (18) in the presence of a thermal bath, or the bosonic Boltzmann operator when elastic collisions are taken into account. An even more general model could take into account both contributions, or include additional semiclassical contributions arising from the fact that the operators $\mathbf{r}$ and $\mathbf{k}$ do not commute.

As a final comment, we may note that there exists analogies between the Bose-Einstein condensation and the inverse cascade process in two-dimensional turbulence where energy accumulates at large scales $(k=0)$ to form a macrovortex [47].

Acknowledgements This study was initiated by one of us (P.H.C) during the 3rd NEXTSigma-Phi Conference in Crete (August 2005). He thanks C. Tsallis, G. Kaniadakis and J. Sommeria for stimulating discussions. 
[1] L. Landau, E. Lifshitz, Statistical Physics (Pergamon Press, 1959).

[2] L. Pitaevskii, S. Stingari, Bose-Einstein Condensation (Clarnedon Press, Oxford, 2003); C.J. Pethick, H. Smith, Bose-Einstein Condensation in Dilute Gases (Cambridge University Press, Cambridge, 2002); F. Dalfovo, S. Giorgini, L. Pitaevskii, S. Stingari, Rev. Mod. Phys. 71, 463 (1999); A. Griffin, D.W. Snoke, S. Stringari, Bose-Einstein Condensation (Cambridge University Press, Cambridge, 1996).

[3] M. H. Anderson, J. R. Ensher, M. R. Matthews, C. E. Wieman, and E. A. Cornell, Science 269, 198 (1995)

[4] C. C. Bradley, C. A. Sackett, J. J. Tollett, and R. G. Hulet, Phys. Rev. Lett. 75, 1687 (1995);

C. C. Bradley, C. A. Sackett, and R. G. Hulet, Phys. Rev. Lett. 78, 985 (1997)

[5] K. B. Davis, M.-O. Mewes, M. R. Andrews, N. J. van Druten, D. S. Durfee, D. M. Kurn, and W. Ketterle, Phys. Rev. Lett. 75, 3969 (1995)

[6] P. Jetzer, Phys. Rep. 220, 163 (1992)

[7] M.S. Turner, Phys. Rep. C 197, 67 (1990).

[8] S.N. Bose, Z. Phys. 26, 178 (1924).

[9] A. Einstein, Preussische akademie der wissenschaften, Phys-math. Klasse, Sitzungsberichte 23, 3 (1925).

[10] L.W. Nordheim, Proc. R. Soc. London A 119, 689 (1928).

[11] F. Bloch, Z. Phys. 52, 555 (1928).

[12] E.A. Uehling and G.E. Uhlenbeck, Phys. Rev. 43, 552 (1933).

[13] D.V. Semikoz, I.I. Tkachev, Phys. Rev. D 55, 489 (1997).

[14] R. Lacaze, P. Lallemand, Y. Pomeau and S. Rica, Physica D 152, 779 (2001).

[15] A.S. Kompaneets, Sov. Phys.-JETP 4, 730 (1957).

[16] G. Kaniadakis and P. Quarati, Phys. Rev. E 48, 4263 (1993); Phys. Rev. E 49, 5103 (1994).

[17] G. Kaniadakis, Physica A 296, 405 (2001); Phys. Lett. A 288, 283 (2001).

[18] L. Bergé and J.J. Rasmussen, Physics Letters A 304, 136 (2002).

[19] G. S. Grest, D. J. Srolovitz and M. P. Anderson, Phys. Rev. B 38, 4752 (1988); M. P. Anderson, G. S. Grest and D. J. Srolovitz, Philos. Mag. B 59, 293 (1989); J. A. Glazier, M. P. Anderson and G. S. Grest, Philos. Mag. B 62, 615 (1990); J. A. Glazier, PhD Thesis (University of 
Chicago, 1989), unpublished.

[20] H. Flyvberg, Phys. Rev. E 47, 4037 (1993); H. Flyvberg and C. Jeppesen, Physica Scripta 38, 49 (1991).

[21] J. Stavans, E. Domany and D. Mukamel, Europhys. Lett. 15, 479 (1991).

[22] C. Sire and S. N. Majumdar, Phys. Rev. Lett. 74, 4321 (1995); Phys. Rev. E 52, 244 (1995).

[23] J. A. Glazier, S. P. Gross and J. Stavans, Phys. Rev. A 36, 306 (1987); J. Stavans and J. A. Glazier, Phys. Rev. Lett. 62, 1318 (1989); J. A. Glazier and J. Stavans, Phys. Rev. A 40, 7398 (1989).

[24] A. J. Bray, Advances in Physics 43, 357 (1994).

[25] F. Liu and G. F. Mazenko, Phys. Rev. B 44, 9185 (1991).

[26] N. Mason, A. N. Pargellis and B. Yurke, Phys. Rev. Lett. 70, 190 (1993); B. Yurke, A. N. Pargellis, S. N. Majumdar and C. Sire, Phys. Rev. E 56, R40 (1997).

[27] P.-H. Chavanis, J. Sommeria and R. Robert, Astrophys. J. 471, 385 (1996).

[28] P.-H. Chavanis and C. Sire, Phys. Rev. E 69, 016116 (2004).

[29] P.-H. Chavanis, C. Rosier and C. Sire, Phys. Rev. E 66, 036105 (2002).

[30] C. Sire and P.-H. Chavanis, Phys. Rev. E 66, 046133 (2002).

[31] C. Sire and P.-H. Chavanis, Phys. Rev. E 69, 066109 (2004).

[32] P.-H. Chavanis and C. Sire, Phys. Rev. E 70, 026115 (2004).

[33] P.-H. Chavanis, M. Ribot, C. Rosier and C. Sire, Banach Center Publ. 66, 103 (2004).

[34] C. Sire and P.-H. Chavanis, Banach Center Publ. 66, 287 (2004) cond-mat/0407397

[35] P.-H. Chavanis and C. Sire, Phys. Rev. E (in press) cond-mat/0504718.

[36] T.D. Frank, Phys. Lett. A 290, 93 (2001).

[37] P.H. Chavanis, Phys. Rev. E 68, 036108 (2003); Physica A 332, 89 (2004); Physica A 340, 57 (2004).

[38] P.H. Chavanis and C. Sire, preprint

[39] V.L. Ginzburg, L.P. Pitaevskii, Zh. Eksp. Teor. Fiz. 34, 1240 (1958); E.P. Gross, J. Math. Phys. 4, 195 (1963).

[40] E. Levich, V. Yakhot, Phys. Rev. B 15, 243 (1977).

[41] H.T.C. Stoof, Phys. Rev. Lett. 66, 3148 (1991); H.T.C. Stoof, Phys. Rev. A 45, 8398 (1992).

[42] H.T.C. Stoof, Phys. Rev. Lett. 78, 768 (1997).

[43] C.W. Gardiner, P. Zoller Phys. Rev. A 55, 2902 (1997). 
[44] C. Connaughton, Y. Pomeau, C. R. Physique 5, 91 (2004).

[45] B.V. Svistunov, J. Moscow Phys. Soc. 1, 373 (1991).

[46] Yu. M. Kagan, B. V. Svistunov, G.V. Shlyapnikov, Zh. Eksp. Teor. Fiz. 101, 528 (1992).

[47] R.H. Kraichnan, Phys. Fluids 10, 1417 (1967).

[48] In a classical Monte-Carlo simulation using local updating (for instance in an Ising spin system, $s_{i} \rightarrow-s_{i}$ with Glauber or Metropolis probabilities), the Monte-Carlo time, as measured as the number of updates, has in fact a physical relevance. In simulations of soap bubbles using the Potts model (a generalization of the Ising model) 19], the Monte-Carlo time is found to be simply proportional to the actual time, and these times can be made equal by a proper choice of $\xi$. Numerically 19], theoretically [20, 21, 22] and in real physical systems [23], the typical area of a bubble is found to grow linearly with time. The out of equilibrium dynamics of the Ising model quenched from high temperature to a temperature below the ferromagnetic critical temperature, leads to a coarsening dynamics where domains of $\uparrow$ and $\downarrow$ spins grow on a scale $L(t)$ 24, 25]. An experiment in nematic liquid crystals exactly mimics this situation [26], where the physical time is found to be indeed proportional to the Monte-Carlo time in simulations. One obtains $L(t) \sim t^{1 / 2}$, and spin temporal correlations are found to have the same functional form [24, 25, 26]. It is clearly comforting and satisfying that the theoretical dynamics modelizing the coupling to a thermal bath leading to equilibrium actually describes the evolution of out of equilibrium physical systems. However in quantum systems, it is not clear at all how to relate the simulation time (number of world lines affected by the MonteCarlo dynamics) to the actual time. 